Forschungszentrum Jülich

Institut für Plasmaphysik

EURATOM Association, Trilateral Euregio Cluster

\title{
Numerical and Analytical Interpretation of Rotation and Radial Electric Fields in Collision Dominated Edge Plasmas
}

Albert Nicolai, Umur Daybelge, André Rogister, Cuma Yarim 




\section{Numerical and Analytical Interpretation of Rotation and Radial Electric Fields in Collision Dominated Edge Plasmas}

Albert Nicolai ${ }^{1}$, Umur Daybelge ${ }^{2}$, André Rogister ${ }^{1}$, Cuma Yarim ${ }^{2}$

\footnotetext{
${ }^{1}$ Institut für Plasmaphysik, Forschungszentrum Jülich $\mathrm{GmbH}$, Euratom Association, Trilateral Euregio Cluster, D-52425 Jülich, Germany

2 Istanbul Technical University, Faculty of Aeronautics and Astronautics, 80626 Maslak, Istanbul, Turkey
} 
Berichte des Forschungszentrums Jülich ; 3958 ISSN 0944-2952

Institut für Plasmaphysik

EURATOM Association, Mitglied im Trilateral Euregio Cluster Jül-3958

Zu beziehen durch: Forschungszentrum Jülich $\mathrm{GmbH} \cdot$ Zentralbibliothek 52425 Jülich - Bundesrepublik Deutschland

02461/61-5220-Telefax: 02461/61-6103·e-mail : zb-publikation@fz-juelich.de 


\title{
Numerical and Analytical Interpretation of Rotation and Radial Electric Fields in Collision Dominated Edge Plasmas
}

\author{
A. Nicolai ${ }^{1}$, U. Daybelge ${ }^{2}$, A. Rogister ${ }^{1}$, C. Yarim $^{2}$ \\ ${ }^{1}$ Institut für Plasmaphysik, Forschungszentrum Jülich GmbH, Euratom / \\ Association, Trilateral Euregio Cluster, D-52425 Jülich, Germany \\ ${ }^{2}$ Istanbul Technical University, Faculty of Aeronautics and Astronautics, \\ 80626 Maslak, Istanbul, Turkey
}

\begin{abstract}
The ambipolarity constraint and the parallel momentum balance equation of neoclassical theory, accounting for finite Larmor radius effects and inertia, allow to describe the radial electric field and the related spin up in collision dominated edge - plamas with steep gradients. Thus they may contribute significantly to the understanding of the L-H transition.

The variation of the toroidal velocity from the last closed magnetic surface up to a position $r$ within the plasma is predicted to be proportional to the integral of the product $u_{\theta} \frac{\partial \ln T}{\partial r}$ i.e., to $\sum_{d}\left[\frac{T_{i}^{\prime 2}}{T_{i}}\right] L_{d}$, if the interaction with the neutral gas can be neglected. The summation is over different radial domains, such as the edge pedestal. $L_{d}$ is the radial extension of the respective domain. The dimensionless parameter $\Lambda=\frac{\nu_{i} q^{2} R^{2}}{\Omega_{i} r L_{T_{i}}}=\frac{\hat{\nu}_{i} a_{i_{p}}}{L_{T_{i}}}\left[\right.$ where $\hat{\nu}_{i}=\frac{q R \nu_{i}}{c_{i}}>0.22$ is the relevant collision parameter and $a_{i_{p}}$ the poloidal ion Larmor radius] characterizes the ratio of the diamagnetic rotation frequency to the heat diffusion rate along magnetic field lines. Conventional neoclassical theory assumes $\Lambda \rightarrow 0$. However, e. g. in ALCATOR C-MOD ohmic H-mode pedestals, $\Lambda$ is sufficiently large that conventional neoclassical results are invalid: it follows from the neoclassical theory that the poloidal velocity decreases below the standard prediction $v_{\text {neo }}=\frac{-1.83 T_{i}^{\prime}}{e B}$ as $\Lambda^{2}$ increases and changes sign for $\Lambda^{2}=\Lambda_{0}^{2}$ (typically $\approx 1-2$ ).

The equations are treated analytically using a linear interpolation for the poloidal velocity, $v_{\theta}\left(\Lambda^{2}\right)$, based on $v_{\theta}\left(\Lambda_{0}^{2}\right)=0$ and on the neoclassical value $v_{\text {neo }}$ for small $\Lambda$. This allows to account for finite $\Lambda$ effects in the just mentioned integration.
\end{abstract}


The equations are also solved numerically (1) to benchmark with a simplified analytical theory with $\Lambda=0$ and vanishing neutral gas density; (2) to compare with the analytical theory accounting for finite $\Lambda$ effects and (3) to explore the parameter space in regions where the analytical theory is not valid, in particular in the cases where the neutral gas density is larger than $10^{14} \mathrm{~m}^{-3}$.

The method resorts to an ODE - solver for the classical momentum balance which is combined with a solver for transcendental equations yielding $v_{\Theta}$.

The results concern the comparison with the analytical solution and the experimental results of the ohmically heated ALCATOR plasma. For $\Lambda=0$ the numerical solution and the analytical one agree exactly. For finite $\Lambda \approx 1$ the deviations are surprisingly small. The toroidal spin up of the ALCATOR plasma, characterized by a very short decay length $L_{\psi}=0.76 \mathrm{~cm}$, is $\approx 40 \frac{\mathrm{km}}{\mathrm{sec}}$. This compares well the measured value of $35 \frac{\mathrm{km}}{\mathrm{sec}}$. The radial electric field profile assumes the characteristic shape and absolute values reported by the DIII-D Group. 


\section{Contents}

1 Introduction 5

2 Ambipolarity and momentum balance in the revisited neoclassical theory

3 Classical Toroidal Momentum Transport

4 Poloidal rotation

5 Description of the plasma rotation by a 2 nd order equation and a transcendental equation

6 Boundary values

7 Halfanalytical solution

8 Analytical integration resorting to a special temperature profile

9 Analytical treatment in the case of finite $\Lambda_{r}$

10 Numerical solution method

11 Friction due to recycled neutrals

12 Neutral injection

13 Results 
13.1 Comparison with the simplified analytical theory of section 8 and the halfanalytical theory of section $7 \ldots \ldots \ldots \ldots 4 . \ldots \ldots$

13.2 Comparison with the approximate analytical theory of section 9 , accounting for finite $\Lambda_{1} \ldots \ldots$. . 45

13.3 General radial profiles of the plasma parameters . 46

13.4 Influence of the neutral gas . . . . . . . . . . . 47

14 Conclusions $\quad 48$

A Temperature and density profiles with $L_{\psi}$ as decay length

70

B Ion - ion collisionality and collision frequency $\quad 73$

$\begin{array}{ll}\text { C Parameter } \Lambda_{1} & 74\end{array}$

$\begin{array}{ll}\text { D Reciprocal time } \eta^{\prime} & 77\end{array}$

$\begin{array}{lll}\text { E Velocity S } & 79\end{array}$

$\begin{array}{lll}\text { F Velocity Q } & 80\end{array}$

G Evaluation of $T_{36} \quad \mathbf{8 3}$

G.1 1st expression . . . . . . . . . . . 83

G.2 2nd expression . . . . . . . . . . . . 83

H Electric drift velocity $\quad 84$

I Temperature profile in Alcator C - MOD 85 


\section{Introduction}

Anomalous plasma transport and the concomitant deterioration of the confinement times of tokamaks much below the neoclassical prediction, in particular if auxiliary heating is applied, are key issues in fusion research.

Therefore the surprising experimental discovery of the transition to a high confinement mode [1] above a certain operating power has evoked considerable interest in improved confinement regimes.

Thus e. g. the $\mathrm{H}$ (high) ([1] - [5]), the VH (very high) [6], the RI (radiation improved) [7] and the ERS (enhanced reversed shear) ([8] - [11]) modes were investigated. The importance of these modes is underlined by the fact that ignition of the International Tokamak Experimental Reactor (ITER) [12] requires an improved confinement regime.

The theoretical models for these transitions and in particular for the L (low) to H transition, ([13] - [17]) are generally speaking not fully selfconsistent. In fact, a model accounting for the interaction of turbulence, MHD - activity and neoclassical effects selfconsistently is out of range.

Here we concentrate on the revisited neoclassical theory ([22] [24]) which, in contrast to the conventional neoclassical theory, allows within the framework of a rigorous analytical approach to calculate the velocity field parallel to the flux surfaces and the ambipolar electric field. This theory is valid in the collision dominated regime which is sometimes, e.g. in [4], encountered in the edge region.

In standard neoclassical theory it is assumed that all macro- 
scopic lengths (connection length $\mathrm{qR}$, minor radius a, and the absolute values of the decay lengths of the ion Temperature $\mathrm{T}$ and ion density $\mathrm{n}, L_{T}=\left\{\frac{\partial \ln T}{\partial r}\right\}^{-1}=T\left(\frac{\partial T}{\partial r}\right)^{-1}$ and $L_{n}=$ $\left\{\frac{\partial \ln n}{\partial r}\right\}^{-1}=n\left(\frac{\partial n}{\partial r}\right)^{-1}$, respectively, are much larger than the ion Larmor radius.

If, however, the decay lengths of the plasma parameters are intermediate between the ion Larmor radius and the connection length, finite Larmor radius (F. L. R.) effects and inertia become important. Therefore standard neoclassical transport theory (predicting e. g. 'automatic' ambipolarity) does not apply to the collision dominated edge region.

Within the framework of the revisited neoclassical theory ([22]$[24],[27])$, the analysis of poloidal or toroidal rotation in a collision dominated toroidal plasma with steep gradients is based on the fluid equations with mass and momentum sources. The revised theory has introduced important modifications into the parallel momentum equation, when the parameter

$$
\Lambda_{1}=\frac{\nu_{i i}}{\Omega_{i}} \frac{q^{2} R^{2}}{L_{T} 4 r}
$$

exceeds $\frac{1}{3}$. $\left(\Omega_{i}, \nu_{i i}\right.$ are the ion gyro- and the ion - ion collision frequency, respectively.)

Since the poloidal velocity is determined in this theory by means of a cubic equation, it has also been speculated that the poloidal velocity as derived by the revisited neoclassical theory would not be unique and under certain conditions allow for bifurcated equilibria.

Poloidal plasma rotation in toroidal systems is related to various instability mechanisms [32]. For example, plasma rotation 
is always accompanied by a radial electric field, whose origin appears to be complicated due to various competing effects, e. g. the friction with the neutral gas and steepening of the temperature profile. It is usually believed that the neoclassical transport should be ambipolar and independent of the radial electric field. However, this requirement is strictly valid only in a plasma without steep gradients as source of toroidal momentum.

Stringer [19] was the first to notice that the resistive diffusion rate in a toroidal plasma can not only be non-ambipolar, but can also be negative for some values of the poloidal rotation velocity.

Rosenbluth and Taylor [20], considered the stability of toroidal diffusion using a fluid model and proved that if the resistivity were the only dissipative mechanism, then even if all plasma deformations were excluded, there could be no stable poloidal rotation velocity.

The rapidly growing poloidal or toroidal rotations, can also be observed as spin-up phenomena, sometimes of unknown origin, in tokamaks.

An interaction between a poloidal or toroidal spin-up and the turbulence driven anomalous transport is also believed to be a likely reason for the aforementioned $\mathrm{L}-\mathrm{H}$ mode transition in tokamaks.

A further consequence of an unstable rotation is that a poloidally asymmetric particle transport may also render the radial electric field unstable [21].

In the here envisaged high collisionality regime with steep gradients only stationary solutions are investigated. The F. L. R. effects enter the ion fluid equations via the gyro - stress ten- 
sor [22]. Since ambipolarity is no longer automatically ensured in these equations, an ambipolarity constraint can be derived allowing to determine the selfconsistent radial electric field $E_{r}$. This constraint together with the momentum balance provide the possibility to compute the parallel ion speed $v_{\|}$and the radial electric field $E_{r}$ or, equivalently, the poloidal velocity component $u_{\Theta}=v_{E}+\frac{B_{\theta}}{B} v_{\|}+v_{n}+\tilde{v}_{T}$ selfconsistently. As mentioned, both equations are nonlinear in $u_{\Theta}$ and $v_{\|}$. ([22]-[24],[27]). (The electric drift speed and the density or temperature related velocities $v_{E}, v_{n}, \tilde{v}_{T}$ are given by $\left.v_{E}=-\frac{E_{r}}{B_{\phi}}, v_{n}=\frac{T}{e B_{\phi}} \frac{\partial \ln n}{\partial r} \tilde{v}_{T}=\frac{T}{e B_{\phi}} \frac{\partial \ln T}{\partial r}\right)$ The momentum source or sinks due to the recycled neutrals and NBI are included in these equations.

The crucial parameter $\Lambda_{1}$ measures the ratio of the contributions arising from perpendicular viscosity to those from the parallel viscosity. To compare with the results of the standard neoclassical theory $\left|\Lambda_{1}\right|$ is chosen to approach zero.

By increasing $\left|\Lambda_{1}\right|$ until 0.5 (as indicated by ASDEX - parameters) the modifications due to the full theory can be investigated. 


\section{Ambipolarity and momentum balance in the revisited neoclassical theory}

The revisited neoclassical theory provides an ambipolarity constraint, which together with the parallel and radial momentum equations allows to account e.g. for the radial electric field, the plasma rotation and the formation of transport barriers [24].

Equations for a two-component plasma, describing the continuity of species $\mathrm{j}$ with sources $S_{j}(\vec{x}, t)$, the momentum balance with friction $\vec{R}_{j}(x, t)$ and momentum input $\vec{S}_{j}^{M}(\vec{x}, t)$, and the energy balance with analogous terms and energy input are

$$
\begin{gathered}
\frac{\partial n_{j}}{\partial t}+\nabla\left(n_{j} \vec{u}_{j}\right)=S_{j} \\
m_{j} n_{j} \frac{d \vec{u}_{j}}{d t}=-\nabla \vec{P}_{j}-\nabla \overrightarrow{\vec{\Pi}}_{j}-e Z_{j} n_{j}\left(\vec{E}_{j}+\frac{1}{c} \vec{u}_{j} \times \vec{B}\right)-\vec{R}_{j}+\vec{S}_{j}^{M}
\end{gathered}
$$

and

$$
\frac{3}{2} n_{j} \frac{d T_{j}}{d t}+P_{j} \nabla u_{j}=-\nabla \vec{q}_{j}-\overrightarrow{\vec{\Pi}}_{j}: \nabla \vec{u}_{j}+R_{j}^{E}+S_{j}^{E}
$$

The viscous tensor is given by

$$
\overrightarrow{\vec{\Pi}}=-\eta_{0} \overrightarrow{\vec{W}}_{0}-\eta_{1}\left(\overrightarrow{\vec{W}}_{1}+4 \overrightarrow{\vec{W}}_{2}\right)+\eta_{3}\left(\overrightarrow{\vec{W}}_{3}+2 \overrightarrow{\vec{W}}_{4}\right)=
$$

The tensors $\overrightarrow{\vec{W}}_{0}, \overrightarrow{\vec{W}}_{1} \overrightarrow{\vec{W}}_{2}, \overrightarrow{\vec{W}}_{3}, \overrightarrow{\vec{W}}_{4}$, accounting for the parallel, perpendicular and gyro stress are given in [18] (recently extended and completed by Mikhailovsky and Tsypin [26]). The terms $S_{j}^{E}$ on the R.H.S. of equation (3) denotes energy sources and losses, whereas $R_{j}^{E}$ denotes terms relating to collisional energy transfer and frictional heating. The correct forms of these 
terms require a kinetic and atomic approach. However, here we shall assume them, as given functions.

The radial electric field satisfies the radial momentum balance

$$
E_{r}+(\vec{U} \times \vec{B})=\frac{1}{e N} \frac{\partial P_{i}}{\partial r}
$$

In the revisited neoclassical theory [1], a plausible ordering inside the separatrix is introduced by means of a small parameter $\mu \approx 0.1$ as

$$
\frac{c_{j}}{q R v_{j}} \approx \frac{L_{\psi}}{r} \approx \frac{r}{q R} \approx \frac{B_{\theta}}{B_{\phi}} \approx \mu
$$

and

$$
\frac{e_{j} V}{T_{j}} \approx \sqrt{\frac{m_{e}}{m_{i}}} \approx \frac{a_{i}}{L_{\psi}} \approx \mu^{2}
$$

where $L_{\psi}$ is the radial scalelength of the temperature, $c_{j}$ and $a_{j}$ are the thermal speed and the gyro radius of the species $\mathrm{j}$, respectively; $\mathrm{V}$ is the loop voltage and $\nu_{j}$ the collision frequency between like particles. Using the magnetic field aligned orthonormal unit vectors $(\hat{p}, \hat{b}, \hat{n})$ in radial, binormal and parallel directions, and the small parameter $\mu$, the velocity of species j can be assumed as,

$$
\begin{gathered}
\hat{p} \vec{U}_{j}=\mu^{6} U_{\psi, j}^{(6)}(\psi, \chi)+\ldots \\
\hat{b} \vec{U}_{j}=\mu^{2} U_{\beta, j}^{(2)}(\psi)+\mu^{3} U_{\beta, j}^{(3)}(\psi, \chi) \ldots \\
\hat{n} \vec{U}_{j}=\mu U_{\|, j}^{(1)}(\psi)+\mu^{2} U_{\|, j}^{(2)}(\psi, \chi) \ldots
\end{gathered}
$$

Assuming that the magnetic field, density, temperature, potential, etc., are independent of the poloidal angle in dominant 
order, these are also expanded in perturbation series. For example, the density and the magnetic field are written as,

$$
N(\psi, \chi)=N^{(0)}(\psi)\left[1+\mu N^{(1)}(\psi, \chi) \ldots\right]
$$

and

$$
B(\psi, \chi)=B^{(0)}(\psi)\left[1+\mu B^{(1)}(\psi, \chi) \ldots\right]
$$

respectively. For a tokamak plasma with circular cross section, also use is made of the toroidal unit vectors $\left(\vec{e}_{r}, \vec{e}_{\theta}, \vec{e}_{\phi}\right)$. Taking toroidal and parallel projections of the momentum equation and averaging them over the magnetic flux surfaces, and imposing the ambipolarity condition, one obtains a pair of coupled nonlinear equations for the toroidal and poloidal ion velocities in terms of other plasma variables, such as temperature, density, and electric field [1]. 


\section{Classical Toroidal Momentum Transport}

The main result of the revisited neoclassical theory [27] is an equation describing the radial transport of toroidal momentum in a collisional subsonic plasma with steep gradients. For large aspect ratio and circular cross - section we get the time - dependent $u_{\phi}$ - equation

$$
\begin{aligned}
& \frac{\partial}{\partial r}\left[\eta_{2}\left(\frac{\partial u_{\phi}}{\partial r}-\frac{0.107 q^{2}}{1+\frac{Q^{2}}{S^{2}}} \frac{\partial \ln T}{\partial r} \frac{B_{\phi}}{B_{\theta}} u_{\theta}\right)\right] \\
= & m_{i} n_{i}\left(\frac{\partial}{\partial t}+\nu_{c x}\right) u_{\phi}-m_{i} n_{i}\left(\dot{M}_{\phi, i}+j_{r} B_{\theta}\right)
\end{aligned}
$$

$\mathrm{Q}$ and $\mathrm{S}$ are given in the appendices $\mathrm{F}$ and $\mathrm{E} . m_{i}, n_{i}$ are the ion mass and ion density, respectively.

The poloidal rotation driven by the temperature gradient seen inside the paranthesis on the right hand side of (5) results from the gyro stress tensor and acts like another source term, i.e., as a toroidal momentum source or sink, depending on the sign of its radial gradient. External momentum sources can be direct, such as fast ions provided by the neutral beam injection (NBI), collisions by alpha particles, or indirect and due to particle sources such as aforementioned charge exchange reactions with cold recycled neutrals. The poloidal rotation velocity $u_{\theta}$, the charge exchange reactions with the recycled cold neutrals, the momentum injection $\dot{M}_{\phi, i}$ by NBI and by a probe generating the radial current density $j_{r}$ determine the toroidal flow velocity $u_{\phi}[25]$. The charge exchange reactions are characterized by the charge exchange frequency

$$
\nu_{c x}=<\sigma v>_{c x} \quad n_{0}
$$


$\langle\sigma v\rangle_{c x}$ is the rate coefficient for charge exchange, and $n_{0}$ the neutral gas density. We define

$$
\begin{aligned}
g & =\frac{u_{\phi}}{v_{T}} \\
h & =\frac{u_{\theta}}{v_{T}}
\end{aligned}
$$

and

$$
x=\frac{r-r_{i n f}}{L_{\psi}}
$$

Here we used the (constant, positive) velocity

$$
v_{T}=\frac{1}{e B_{\phi}} \frac{T_{i n f}}{L_{\psi}}
$$

$T_{\text {inf }}$ is the temperature at the inflection point $P_{\text {inf }}$ of the temperature profile (Fig. 1). The inflection point has the radius $r_{\text {inf }}$ and is defined as the locus of vanishing curvature of the temperature profile. $L_{\psi}$ is the temperature scale length at $P_{\text {inf }}$ in absolute value (appendix A). We get

$$
\begin{gathered}
\frac{v_{T}}{L_{\psi}} \frac{\partial}{\partial x}\left[\eta_{2}\left(\frac{\partial g}{\partial x}-\frac{0.107 q^{2}}{1+\frac{Q^{2}}{S^{2}}} \frac{\partial \ln T}{\partial x} \frac{B_{\phi}}{B_{\theta}} h\right)\right] \\
=m_{i} n_{i n f} \hat{n} v_{T}\left(\frac{\partial}{\partial t}+\nu_{c x}\right) g-m_{i} n_{i n f} \hat{n}\left(\dot{M}_{\phi, i}+j_{r} B_{\theta}\right)
\end{gathered}
$$

$n_{\text {inf }}$ is the density at the inflection point and $\hat{n}$ is defined by $n_{i}=\hat{n} n_{i n f}$. The characteristic time $t_{c}$ is given by

$$
t_{c}^{-1}=\frac{1}{m_{i} n_{i n f} L_{\psi}^{2}} \eta_{2, i n f}
$$


We introduce this time by multiplying with $\frac{1}{m_{i} n_{i n f} L_{\psi \psi}^{2}}$. With the decomposition

$$
\eta_{2}=\eta_{2, i n f} \hat{\eta}_{2}
$$

we can write

$$
\begin{gathered}
\frac{\eta_{2, i n f}}{m_{i} n_{i n f} L_{\psi}^{2}}\left(\frac{\partial}{\partial x}\left[\hat{\eta}_{2}\left(\frac{\partial g}{\partial x}-\frac{0.107 q^{2}}{1+\frac{Q^{2}}{S^{2}}} \frac{\partial \ln T}{\partial x} \frac{B_{\phi}}{B_{\theta}} h\right)\right]\right. \\
\left.=\hat{n}\left(\frac{\partial}{\partial t}+\nu_{c x}\right) g-\frac{\hat{n} \dot{M}_{\phi, i}}{v_{T}}\right]+\frac{J_{r} B_{\theta}}{m_{i} v_{T} n_{i n f}}
\end{gathered}
$$

We define

$$
G=\frac{\partial g}{\partial x}-\frac{0.107 q^{2}}{1+\frac{Q^{2}}{S^{2}}} \frac{\partial \ln T}{\partial x} \frac{B_{\phi}}{B_{\theta}} h
$$

and get

$$
\begin{gathered}
\frac{\eta_{2, i n f}}{m_{i} n_{i n f} L_{\psi}^{2}} \frac{\partial}{\partial x}\left(\hat{\eta}_{2} G\right) \\
=\hat{n}\left(\frac{\partial}{\partial t}+\nu_{c x}\right) g-\frac{\hat{n} \dot{M}_{\phi, i}}{v_{T}}+\frac{j_{r} B_{\theta}}{m_{i} v_{T} n_{i n f}}
\end{gathered}
$$

By differentiating we obtain

$$
\begin{gathered}
\frac{\eta_{2, i n f}}{m_{i} n_{i n f} L_{\psi}^{2}}\left(G \frac{\partial}{\partial x} \hat{\eta}_{2}+\hat{\eta}_{2} \frac{\partial}{\partial x} G\right) \\
=\hat{n}_{i}\left(\frac{\partial}{\partial t}+\nu_{c x}\right) g-\frac{\hat{n} \dot{M}_{\phi, i}}{v_{T}}+\frac{J_{r} B_{\theta}}{m_{i} v_{T} n_{i n f}}
\end{gathered}
$$

or

$$
\begin{aligned}
& \frac{\eta_{2, i n f}}{m_{i} n_{i n f} L_{\psi}^{2}} \hat{\eta}_{2}\left(G \frac{\partial}{\partial x} \ln \left(\hat{\eta}_{2}\right)+\frac{\partial}{\partial x} G\right) \\
= & \hat{n}\left(\frac{\partial}{\partial t}+\nu_{c x}\right) g-\frac{\hat{n} \dot{M}_{\phi, i}}{v_{T}}+\frac{J_{r} B_{\theta}}{m_{i} v_{T} n_{i n f}}
\end{aligned}
$$


Furthermore we get

$$
\begin{gathered}
\frac{\eta_{2, i n f}}{m_{i} n_{i n f} L_{\psi}^{2}} \hat{\eta}_{2} \frac{\partial}{\partial x} G=\hat{n}\left(\frac{\partial}{\partial t}+\nu_{c x}\right) g-\frac{\hat{n} \dot{M}_{\phi, i}}{v_{T}}+ \\
\frac{J_{r} B_{\theta}}{m_{i} v_{T} n_{i n f}}-\frac{1}{m_{i} n_{\text {inf }} L_{\psi}^{2}} \eta_{2} G \frac{\partial}{\partial x} \ln \left(\eta_{2}\right)
\end{gathered}
$$

and

$$
\begin{gathered}
\frac{\partial}{\partial x} G=\frac{m_{i} n_{i n f} L_{\psi}^{2}}{\eta_{2, i n f} \hat{\eta}_{2}}=\left[\hat{n}\left(\frac{\partial}{\partial t}+\nu_{c x}\right) g-\frac{\hat{n} \dot{M}_{\phi, i}}{v_{T}}+\right. \\
\left.\frac{J_{r} B_{\theta}}{m_{i} v_{T} n_{i n f}}-\frac{\eta_{2, i n f}}{m_{i} n_{\text {inf }} L_{\psi}^{2}} \hat{\eta}_{2} G \frac{\partial}{\partial x} \ln \left(\eta_{2}\right)\right]
\end{gathered}
$$

The coefficient $\frac{1}{m_{i} n_{\text {inf }} L_{\psi}^{2}} \eta_{2, i n f}=\eta^{\prime}$ containing the viscosity can be rewritten as

$$
\eta^{\prime}=\frac{\frac{6}{5} n_{i n f} k T_{i n f} \tau_{i i}}{\Omega_{i}^{2} \tau_{i i}^{2} m_{i} n_{i n f} L_{\psi}^{2}}
$$

We use $k T_{\text {inf }}=m_{i} c_{\text {inf }}^{2} \hat{T}$, where $c_{\text {inf }}$ is the thermal speed at the inflection point and get

$$
\eta^{\prime}=\frac{\frac{6}{5} \hat{T} c_{i n f}^{2}}{\Omega_{i}^{2} \tau_{i i} L_{\psi}^{2}}
$$

or (appendix D)

$$
\eta^{\prime}=8.5710^{-7} \frac{a_{i, i n f}^{2}}{L_{\psi}^{2}} \frac{n_{i n f} Z_{e f f_{i n f}}}{T_{i n f}^{\frac{3}{2}}} \sqrt{\frac{1}{A}}
$$

$a_{i, \text { inf }}$ is the ion Larmorradius at the inflection point.

We assume

$$
Z_{e f f_{i}}=Z_{e f f_{i n f}} \hat{T}^{Z}
$$


where $\mathrm{Z}$ is a constant. Then the radial dependence of $\eta_{2}$ is given by (one $\hat{T}$ is cancelled)

$$
\hat{\eta}_{2}=\hat{n} \hat{T}^{\left(-\frac{1}{2}+Z\right)}
$$

We get the derivative

$$
\frac{\partial}{\partial x}\left(\hat{\eta}_{2}\right)=\hat{T}^{-\frac{1}{2}+Z} \frac{\partial \hat{n}}{\partial x}+n\left(-\frac{1}{2}+Z\right) \hat{T}^{-\frac{3}{2}+Z} \frac{\partial \hat{T}}{\partial x}
$$

The logarithmic derivative is

$$
\frac{\partial}{\partial x} \ln \left(\eta_{2}\right)=\frac{\partial}{\partial x} \ln \left(\hat{\eta}_{2}\right)=\frac{\hat{T}^{-\frac{1}{2}+Z} \frac{\partial \hat{n}}{\partial x}+\hat{n}\left(-\frac{1}{2}+Z\right) \hat{T}^{-\frac{3}{2}+Z} \frac{\partial \hat{T}}{\partial x}}{\hat{n} \hat{T}^{-\frac{1}{2}+Z}}
$$

or

$$
\frac{\partial}{\partial x} \ln \left(\eta_{2}\right)=\frac{1}{\hat{n}} \frac{\partial \hat{n}}{\partial x}+\frac{\left(-\frac{1}{2}+Z\right)}{\hat{T}} \frac{\partial \hat{T}}{\partial x}
$$




\section{Poloidal rotation}

Using the ambipolarity condition and the extended forms of the stress tensors in the parallel momentum equation, one can cancel the time derivative and the source terms. The result - in the lowest order - is a nonlinear equation between the radial derivatives of the poloidal and toroidal plasma velocities. For large aspect ratio and circular cross - section it can be written as [25]

$$
\begin{gathered}
u_{\theta}+\frac{1.833}{e B_{\phi}} \frac{\partial T}{\partial r}=0.36 \frac{\eta_{1}}{\eta_{0}\left(1+\frac{Q^{2}}{S^{2}}\right)} q^{2} R^{2} \frac{e B_{\phi}}{T_{i}} \frac{\partial \ln T}{\partial r} \\
\left\{\frac{T}{e B_{\theta}} \frac{\partial u_{\phi}}{\partial r}+\frac{1}{2} u_{\phi}^{2}\right. \\
-u_{\phi} \frac{B_{\phi}}{B_{\theta}}\left(u_{\theta}-\frac{T}{e B_{\phi}} \frac{\partial \ln \left(T n_{i}^{2}\right)}{\partial r}\right)+ \\
\left.1.9 \frac{B_{\phi}^{2}}{B_{\theta}^{2}}\left[u_{\theta}-0.8 \frac{T}{e B_{\phi}} \frac{\partial \ln \left(T n_{i}^{1.6}\right)}{\partial r}\right]^{2}\right\}
\end{gathered}
$$

This may be rewritten as

$$
\begin{gathered}
u_{\theta}+\frac{1.833}{e B_{\phi}} \frac{\partial T}{\partial r}= \\
T_{36}\left\{\frac{T_{i}}{e B_{\theta}} \frac{\partial u_{\phi}}{\partial r}+\frac{1}{2} u_{\phi}^{2}\right. \\
-u_{\phi} \frac{B_{\phi}}{B_{\theta}}\left(u_{\theta}-\frac{T_{i}}{e B_{\phi}} \frac{\partial \ln \left(T n_{i}^{2}\right)}{\partial r}\right)+ \\
\left.1.9 \frac{B_{\phi}^{2}}{B_{\theta}^{2}}\left[u_{\theta}-0.8 \frac{T}{e B_{\phi}} \frac{\partial \ln \left(T n_{i}^{1.6}\right)}{\partial r}\right]^{2}\right\}
\end{gathered}
$$


$T_{36}$ is given by

$$
T_{36}=0.36 \frac{\eta_{1}}{\eta_{0}\left(1+\frac{Q^{2}}{S^{2}}\right)} q^{2} R^{2} \frac{e B_{\phi}}{T} \frac{\partial \ln T}{\partial r}
$$

We use $v_{T}=\frac{1}{e B_{\phi}} \frac{T_{i n f}}{L_{\psi}}$ and get the following expression for the LHS of the preceding equation

$$
u_{\theta}+\frac{1.833}{e B_{\phi}} \frac{\partial T}{\partial r}=v_{T}\left(h+1.833 \frac{\partial \hat{T}}{\partial x}\right)
$$

$T_{36}$ may be rewritten as

$$
\begin{gathered}
T_{36}=0.36 \frac{\eta_{1}}{\eta_{0}\left(1+\frac{Q^{2}}{S^{2}}\right)} q^{2} R^{2} \frac{e B_{\phi}}{T} \frac{\partial \ln T}{\partial r}= \\
0.36 \frac{\eta_{1}}{\eta_{0}\left(1+\frac{Q^{2}}{S^{2}}\right)} q^{2} \frac{e B_{\phi} L_{\psi}}{T} \frac{R}{L_{\psi}} \frac{R}{L_{\psi}} L_{\psi} \frac{\partial \ln T}{\partial r} \\
\quad=0.36 \frac{\eta_{1}}{\eta_{0}\left(1+\frac{Q^{2}}{S^{2}}\right)} q^{2} \frac{1}{v_{T} \hat{T}} \frac{R^{2}}{L_{\psi}^{2}} \frac{\partial \ln T}{\partial x}
\end{gathered}
$$

The viscosities $\eta_{1}, \eta_{0}$ can be written as [18]

$$
\begin{gathered}
\eta_{1}=\frac{\frac{6}{5} n_{i} T \tau_{i i}}{\Omega_{i}^{2} \tau_{i i}^{2}} \\
\eta_{0}=\frac{0.96 n_{i} T \nu_{i i}}{\nu_{i i}^{2}}
\end{gathered}
$$

For the ratio $\frac{\eta_{1}}{\eta_{0}}$ we get

$$
\frac{\eta_{1}}{\eta_{0}}=\frac{\frac{\frac{6}{5} n_{i} T \nu_{i i}}{\Omega_{i}^{2}}}{\frac{0.96 P_{i i} \nu_{i i}}{\nu_{i i}^{2}}}=\frac{6}{5 \cdot 0.96} \frac{\nu_{i i}^{2}}{\Omega_{i}^{2}}
$$


Therefore the factor $T_{36}$ in the preceding term becomes

$$
\begin{gathered}
T_{36}=0.36 \frac{\eta_{1}}{\eta_{0}\left(1+\frac{Q^{2}}{S^{2}}\right)} q^{2} \frac{1}{v_{T} \hat{T}} \frac{R^{2}}{L_{\psi}^{2}} \frac{\partial \ln T}{\partial x} \\
=0.36 \frac{6}{50.96} \frac{\nu_{i i}^{2}}{\Omega_{i}^{2}} \frac{1}{\left(1+\frac{Q^{2}}{S^{2}}\right)} q^{2} \frac{1}{v_{T} \hat{T}} \frac{R^{2}}{L_{\psi}^{2}} \frac{\partial \ln T}{\partial x} \\
=0.45 \frac{\nu_{i i}^{2}}{\Omega_{i}^{2}} \frac{1}{\left(1+\frac{Q^{2}}{S^{2}}\right)} q^{2} \frac{1}{v_{T} \hat{T}} \frac{R^{2}}{L_{\psi}^{2}} \frac{\partial \ln T}{\partial x}
\end{gathered}
$$

The other terms of equation (24) are

$$
\begin{gathered}
\frac{T}{e B_{\theta}} \frac{\partial u_{\phi}}{\partial r}=\frac{B_{\phi}}{B_{\theta}} \frac{T}{e B_{\phi} L_{\psi}} \frac{\partial u_{\phi}}{\partial x}=\frac{B_{\phi}}{B_{\theta}} v_{T}^{2} \hat{T} \frac{\partial g}{\partial x} \\
-u_{\phi} \frac{B_{\phi}}{B_{\theta}}\left(u_{\theta}-\frac{T}{e B_{\phi}} \frac{\partial \ln \left(T n_{i}^{2}\right)}{\partial r}\right)= \\
-v_{T}^{2} g \frac{B_{\phi}}{B_{\theta}}\left(h-\hat{T} \frac{\partial \ln \left(T n_{i}^{2}\right)}{\partial x}\right)
\end{gathered}
$$

and

$$
\begin{gathered}
1.9 \frac{B_{\phi}^{2}}{B_{\theta}^{2}}\left[u_{\theta}-0.8 \frac{T_{i}}{e B_{\phi}} \frac{\partial \ln \left(T n_{i}^{1.6}\right)}{\partial r}\right]^{2}= \\
1.9 \frac{B_{\phi}^{2}}{B_{\theta}^{2}} v_{T}^{2}\left[h-0.8 \hat{T} \frac{\partial \ln \left(T n_{i}^{1.6}\right)}{\partial x}\right]^{2}
\end{gathered}
$$

Thus equation (24) can be written as

$$
\begin{gathered}
v_{T}\left(h+1.833 \frac{\partial \hat{T}}{\partial x}\right)= \\
T_{36}\left\{\frac{B_{\phi}}{B_{\theta}} v_{T}^{2} \hat{T} \frac{\partial g}{\partial x}+\frac{1}{2} v_{T}^{2} g^{2}\right.
\end{gathered}
$$




$$
\begin{gathered}
-v_{T}^{2} g \frac{B_{\phi}}{B_{\theta}}\left(h-\hat{T} \frac{\partial \ln \left(T n_{i}^{2}\right)}{\partial x}\right) \\
\left.+1.9 \frac{B_{\phi}^{2}}{B_{\theta}^{2}} v_{T}^{2}\left[h-0.8 \hat{T} \frac{\partial \ln \left(T n_{i}^{1.6}\right)}{\partial x}\right]^{2}\right\}
\end{gathered}
$$

We multiply with $\hat{T} v_{T}$ and cancel $v_{T}^{2}$

$$
\begin{gathered}
\left(h+1.833 \frac{\partial \hat{T}}{\partial x}\right) \hat{T}= \\
C_{\eta}\left\{\frac{B_{\phi}}{B_{\theta}} \hat{T} \frac{\partial g}{\partial x}+\frac{1}{2} g^{2}\right. \\
-g \frac{B_{\phi}}{B_{\theta}}\left(h-\hat{T} \frac{\partial \ln \left(T n_{i}^{2}\right)}{\partial x}\right) \\
\left.+1.9 \frac{B_{\phi}^{2}}{B_{\theta}^{2}}\left[h-0.8 \hat{T} \frac{\partial \ln \left(T n_{i}^{1.6}\right)}{\partial x}\right]^{2}\right\}
\end{gathered}
$$

$C_{\eta}$ is defined by

$$
\begin{gathered}
C_{\eta}=v_{T} \hat{T} T_{36}= \\
0.45 q^{2} \frac{R^{2}}{L_{\psi}^{2}} \frac{\nu_{i i}\left(n_{\text {inf }}, T_{\text {inf }}\right)^{2}}{\Omega_{i}^{2}}\left(\hat{T}^{-\frac{3}{2}} T^{Z} \hat{n}\right)^{2} \frac{1}{\left(1+\frac{Q^{2}}{S^{2}}\right)} \frac{\partial \ln T}{\partial x}
\end{gathered}
$$




\section{Description of the plasma rotation by a 2 nd order equation and a transcendental equa- tion}

We start with equation (7) for $\mathrm{g}$

$$
\begin{gathered}
\frac{v_{T}}{L_{\psi}^{2}} \frac{\partial}{\partial x}\left[\eta_{2}\left(\frac{\partial g}{\partial x}-\frac{0.107 q^{2}}{1+\frac{Q^{2}}{S^{2}}} \frac{\partial \ln T}{\partial x} \frac{B_{\phi}}{B_{\theta}} h\right)\right] \\
=m_{i} n_{i n f} \hat{n} v_{T}\left(\frac{\partial}{\partial t}+\nu_{c x}\right) g-m_{i} n_{\text {inf }} \hat{n} \dot{M}_{\phi, i}+J_{r} B_{\theta}
\end{gathered}
$$

$n_{\text {inf }}$ is the density at the inflection point and $\hat{n}$ is defined by $n_{i}=\hat{n} n_{i n f}$. To get the characteristic time we multiply - as before - by $\frac{1}{m_{i} n_{i n f} v_{T}}$ and get using $\eta_{2}=\eta_{2, i n f} \hat{\eta}_{2}$

$$
\begin{gathered}
\frac{\eta_{2, i n f}}{m_{i} n_{i n f} L_{\psi}^{2}}\left(\frac{\partial}{\partial x}\left[\hat{\eta}_{2}\left(\frac{\partial g}{\partial x}-\frac{0.107 q^{2}}{1+\frac{Q^{2}}{S^{2}}} \frac{\partial \ln T}{\partial x} \frac{B_{\phi}}{B_{\theta}} h\right)\right]\right. \\
\left.=\hat{n}\left(\frac{\partial}{\partial t}+\nu_{c x}\right) g-\frac{\hat{n} \dot{M}_{\phi, i}}{v_{T}}\right]+\frac{J_{r} B_{\theta}}{m_{i} v_{T} n_{i n f}}
\end{gathered}
$$

The characteristic time $t_{c}$ is given by

$$
t_{c}^{-1}=\frac{1}{m_{i} n_{\text {inf }} L_{\psi}^{2}} \eta_{2, i n f}
$$

We define

$$
G=\frac{\partial g}{\partial x}-\frac{0.107 q^{2}}{1+\frac{Q^{2}}{S^{2}}} \frac{\partial \ln T}{\partial x} \frac{B_{\phi}}{B_{\theta}} h
$$

and get the first order equation

$$
\frac{\eta_{2, i n f}}{m_{i} n_{i n f} L_{\psi}^{2}} \frac{\partial}{\partial x}\left(\hat{\eta}_{2} G\right)
$$




$$
=\hat{n}\left(\frac{\partial}{\partial t}+\nu_{c x}\right) g-\frac{\hat{n}_{i} \dot{M}_{\phi, i}}{v_{T}}+\frac{J_{r} B_{\theta}}{m_{i} v_{T} n_{\text {inf }}}
$$

By differentiating we obtain

$$
\begin{gathered}
\frac{\eta_{2, i n f}}{m_{i} n_{i n f} L_{\psi}^{2}}\left(G \frac{\partial}{\partial x} \hat{\eta}_{2}+\hat{\eta}_{2} \frac{\partial}{\partial x} G\right) \\
=\hat{n}\left(\frac{\partial}{\partial t}+\nu_{c x}\right) g-\frac{\hat{n} \dot{M}_{\phi, i}}{v_{T}}+\frac{J_{r} B_{\theta}}{m_{i} v_{T} n_{i n f}}
\end{gathered}
$$

or, resolving with respect to $\frac{\partial}{\partial x} G$ we get

$$
\begin{gathered}
\frac{\partial}{\partial x} G=\frac{m_{i} n_{i n f} L_{\psi}^{2}}{\eta_{2, i n f} \hat{\eta}_{2}}\left[\hat{n}\left(\frac{\partial}{\partial t}+\nu_{c x}\right) g-\frac{\hat{n} \dot{M}_{\phi, i}}{v_{T}}+\right. \\
\left.\frac{J_{r} B_{\theta}}{m_{i} v_{T} n_{\text {inf }}}-\frac{\eta_{2, i n f}}{m_{i} n_{\text {inf }} L_{\psi}^{2}} \hat{\eta}_{2} G \frac{\partial}{\partial x} \ln \left(\hat{\eta}_{2}\right)\right]
\end{gathered}
$$

In the last term the factor $\hat{\eta}_{2}$ appears because the 'In' was introduced. The equation can be written in the form (omitting $\left.\frac{\partial g}{\partial t}\right)$

$$
\frac{\partial}{\partial x} G=T_{C X}+T_{N B}+T_{P r}-G \frac{\partial}{\partial x} \ln \left(\hat{\eta}_{2}\right)
$$

Here

$$
\begin{gathered}
T_{C X}=t_{c} \frac{\hat{n}}{\hat{\eta}_{2}} \nu_{c x} g=t_{c} \hat{T}^{0.5-Z} \nu_{c x} g \\
T_{N B}=t_{c} \frac{\hat{n}}{\hat{\eta}_{2} v_{T}} \dot{M}_{\phi, i}
\end{gathered}
$$

and

$$
T_{P r}=t_{c} \frac{J_{r} B_{\theta}}{m_{i} v_{T} n_{\text {inf }} \hat{\eta}_{2}}
$$

account for the friction caused by the neutral gas, the momentum source due to neutral beam injection and the momentum source due to a probe, respectively. 


\section{Boundary values}

The solution of the preceding equations is strongly influenced by the the boundary values which are therefore discussed in the following.

Firstly we assume a symmetric streaming of the scrape-off plasma into the divertor. This yields as boundary value $\mathrm{h}\left(r=r_{s}\right)=0$. We note that this assumption determines the value of $\Lambda\left(r=r_{s}\right)$ at the boundary. Via the temperature profile we then get the radius $r_{s}$. Thus the condition $\mathrm{h}\left(r=r_{s}\right)=0$ is not a boundary condition for the differential equations, but determines the radial extension.

Secondly we assume $g\left(r=r_{s}\right)=0$ because of the absence of momentum sources such as neutral beam injection (NBI) and because neutrals may be reducing $g\left(r=r_{s}\right)$ to a low value. (We note, however, that at JET [33] and JT60, i. e. devices with strong NBI, a considerable spin up at the separatrix had been observed.)

The analytical or numerical integration can start at the inside $(\mathrm{r}=0)$ or at the outside $\left(r=r_{s}\right)$.

For integrating from the outside to the inside - what may be considered as the 'natural' procedure - $g$ and $\frac{\partial g}{\partial x}$ are prescribed and $\mathrm{G}$ follows from equation (38). If the neutral gas can be neglected, NBI and the probe are switched of, the boundary condition is $\mathrm{G}=0$.

If the neutral gas density is finite as in chapter 13, the derivative $\left.\frac{\partial g}{\partial x}\right|_{r=r_{s}}$ must be adjusted such that $\left.\frac{\partial g}{\partial x}\right|_{r=0}=0$ ('shooting method').

For integrating from the inside to the outside $g$ and $G$ are pre- 
scribed. From equation (38) follows $G(x=-\infty)=0$. Now $\mathrm{g}(r=0)$ must be adjusted to hit a prescribed boundary value $\mathrm{g}\left(r=r_{s}\right)$ at the outside. 


\section{Halfanalytical solution}

We envisage the equation

$$
\begin{aligned}
& \frac{v_{T}}{L_{\psi}} \frac{\partial}{\partial x}\left[\eta_{2}\left(\frac{\partial g}{\partial x}-\frac{0.107 q^{2}}{1+\frac{Q^{2}}{S^{2}}} \frac{\partial \ln T}{\partial x} \frac{B_{\phi}}{B_{\theta}} h\right)\right] \\
= & m_{i} N_{i n f} \hat{n} v_{T}\left(\frac{\partial}{\partial t}+\nu_{c x}\right) g-m_{i} N_{i n f} \hat{n} \dot{M}_{\phi, i}
\end{aligned}
$$

Considering stationarity and neglecting injection, probe current and the neutral gas yields

$$
\frac{\partial}{\partial x}\left[\eta_{2}\left(\frac{\partial g}{\partial x}-\frac{0.107 q^{2}}{1+\frac{Q^{2}}{S^{2}}} \frac{\partial \ln T}{\partial x} \frac{B_{\phi}}{B_{\theta}} h\right)\right]=0
$$

Integrating radially we get

$$
\eta_{2}\left(\frac{\partial g}{\partial x}-\frac{0.107 q^{2}}{1+\frac{Q^{2}}{S^{2}}} \frac{\partial \ln T}{\partial x} \frac{B_{\phi}}{B_{\theta}} h\right)=\text { Const }
$$

In the interior the term $\frac{0.107 q^{2}}{1+\frac{Q^{2}}{S^{2}}} \frac{\partial \ln T}{\partial x} \frac{B_{\phi}}{B_{\theta}} h$ vanishes, therefore

$$
\left.\eta_{2} \frac{\partial g}{\partial x}\right|_{x=-\infty}=\text { Const }
$$

In an infinitesimally small volume around the magnetic axis the thermodynamic quantities are constant because of the nesting of the flux surfaces around this axis. Therefore we get for the preceding equation Const $=0$. We integrate radially again and get the general solution

$$
g-g_{0}=\int_{x=-\infty}^{x} d x^{\prime}\left[\frac{0.107 q^{2}}{\left(1+\frac{Q^{2}}{S^{2}}\right)} \frac{\partial \ln T}{\partial x} \frac{B_{\phi}}{B_{\theta}} h\right]
$$


$g_{0}$ is the boundary value of $\mathrm{g}$ at $\mathrm{r}=0$ or $x=-\infty$ (concerning the boundary values also see chapter 6 ).

We consider the neoclassical approximation of equation (24) for $C_{\eta}=0$

$$
h=-1.833 \frac{\partial \hat{T}}{\partial x}
$$

With this equation we get

$$
g-g_{0}=\int_{x=-\infty}^{x} d x^{\prime}\left[\frac{0.107 q^{2}}{\left(1+\frac{Q^{2}}{S^{2}}\right)} \frac{\partial \ln T}{\partial x} \frac{B_{\phi}}{B_{\theta}}\left(-1.833 \frac{\partial T}{\partial x}\right)\right]
$$

or

$$
\left.g-g_{0}=\int_{x=-\infty}^{x} d x^{\prime} \frac{0.107 q^{2}}{\left(1+\frac{Q^{2}}{S^{2}}\right)} \frac{B_{\phi}}{B_{\theta}}\left(\frac{-1.833}{\hat{T}}\left[\frac{\partial \hat{T}}{\partial x}\right]^{2}\right)\right]
$$




\section{Analytical integration resorting to a special temperature profile}

We evaluate equation (50) in the case of the profile

$$
T=T_{i n f}\left(1-\tanh \frac{r-r_{i n f}}{\Delta_{T}}\right)=T_{i n f}(1-\tanh (x))
$$

which is normalized to $T_{\text {inf }}$, the temperature at the inflection point $r=r_{\text {inf }}$ (Fig. 1). The profile (51) stands for the pedestal at the boundary and thus contains the main property leading to the spin up of the plasma. With the derivatives

$$
\frac{\partial \ln T}{\partial x}=-\frac{1}{(1-\tanh (x)) \operatorname{csh}^{2}(x)}
$$

and

$$
\frac{\partial T}{\partial x}=-\frac{1}{\operatorname{csh}^{2}(x)}
$$

we get

$$
\begin{gathered}
g\left(x^{\prime}\right)-g_{0}=\int_{-\infty}^{x^{\prime}} d x \\
\left(1.833 \frac{0.107 q^{2}}{\left(1+\frac{Q^{2}}{S^{2}}\right)} \frac{1}{(1-\tanh (x)) \operatorname{csh}^{2}(x)} \frac{B_{\phi}}{B_{\theta}} \times\right.
\end{gathered}
$$

We use the identities

$$
\begin{gathered}
\frac{1}{(1-\tanh (x)) \operatorname{csh}^{4}(x)}=\frac{\left(1-\tanh (x)^{2}\right)^{2}}{(1-\tanh (x))}= \\
\frac{(1-\tanh (x))^{2}(1+\tanh (x))^{2}}{(1-\tanh (x))}= \\
(1-\tanh (x))(1+\tanh (x))^{2}
\end{gathered}
$$


and

$$
\begin{gathered}
\frac{1}{(1-\tanh (x)) \operatorname{csh}^{4}(x)}= \\
=\left(1-\tanh ^{2}(x)\right)(1+\tanh (x))=\frac{1+\tanh (x)}{\operatorname{csh}^{2}(x)}
\end{gathered}
$$

This yields

$$
\begin{gathered}
g\left(x^{\prime}\right)-g_{0}=\frac{0.107 q^{2}}{\left(1+\frac{Q^{2}}{S^{2}}\right)} \frac{B_{\phi}}{B_{\theta}} \times \\
\left(\left.1.833\left(\tanh (x)+0.5 \tanh ^{2}(x)\right)\right|_{-\infty} ^{x^{\prime}}\right.
\end{gathered}
$$




\section{Analytical treatment in the case of finite $\Lambda_{r}$}

The $u_{\phi}$ - equation is in the case of vanishing neutral gas density given by

$$
\frac{\partial g}{\partial x}=-\frac{0.107 q^{2}}{1+\frac{Q^{2}}{S^{2}}} \frac{\partial \ln T}{\partial x} \frac{B_{\phi}}{B_{\theta}} h
$$

With $h=h^{\prime} \frac{\partial \hat{T}}{\partial x}$ and $g=g^{\prime} \frac{B_{\phi}}{B_{\theta}}$ we obtain

$$
\frac{\partial g^{\prime}}{\partial x}=-\frac{0.107 q^{2}}{1+\frac{Q^{2}}{S^{2}}} \frac{\partial \ln T}{\partial x} h^{\prime} \frac{\partial \hat{T}}{\partial x}
$$

The $u_{\theta}$ - equation can be written as

$$
\begin{gathered}
\frac{\tilde{v}_{T}}{\frac{\partial \hat{T}}{\partial x}}\left(h+1.833 \frac{\partial \hat{T}}{\partial x}\right)=\tilde{v}_{T}\left(h^{\prime}+1.833\right)= \\
T_{36}\left\{\frac{B_{\phi}}{B_{\theta}} \frac{\tilde{v}_{T}^{2}}{\left[\frac{\partial \hat{T}}{\partial x}\right]^{2}} \hat{T} \frac{\partial g}{\partial x}+\frac{1}{2} \frac{\tilde{v}_{T}^{2}}{\left[\frac{\partial \hat{T}}{\partial x}\right]^{2}} g^{2}\right. \\
-\frac{\tilde{v}_{T}^{2}}{\left[\frac{\partial \hat{T}}{\partial x}\right]^{2}} g \frac{B_{\phi}}{B_{\theta}}\left(h-\hat{T} \frac{\partial \ln \left(T n_{i}^{2}\right)}{\partial x}\right) \\
\left.+1.9 \frac{B_{\phi}^{2}}{B_{\theta}^{2}} \frac{\tilde{v}_{T}^{2}}{\left[\frac{\partial \hat{T}}{\partial x}\right]^{2}}\left[h-0.8 \hat{T} \frac{\partial \ln \left(T n_{i}^{1.6}\right)}{\partial x}\right]^{2}\right\}
\end{gathered}
$$

or

$$
\begin{gathered}
\tilde{v}_{T}\left(h^{\prime}+1.833\right)= \\
T_{36}\left[\frac{B_{\phi}}{B_{\theta}}\right]^{2}\left\{\frac{\tilde{v}_{T}^{2}}{\left[\frac{\partial \hat{T}}{\partial x}\right]^{2}} \hat{T} \frac{B_{\theta}}{B_{\phi}} \frac{\partial g}{\partial x}+\frac{1}{2} \frac{\tilde{v}_{T}^{2}}{\left[\frac{\partial \hat{T}}{\partial x}\right]^{2}}\left[\frac{B_{\theta}}{B_{\phi}}\right]^{2} g^{2}\right. \\
-\frac{\tilde{v}_{T}^{2}}{\left[\frac{\partial \hat{T}}{\partial x}\right]^{2}} g \frac{B_{\theta}}{B_{\phi}}\left(h-\hat{T} \frac{\partial \ln \left(T n_{i}^{2}\right)}{\partial x}\right)
\end{gathered}
$$




$$
\left.+1.9 \frac{\tilde{v}_{T}^{2}}{\left[\frac{\partial \hat{T}}{\partial x}\right]^{2}}\left[h-0.8 \hat{T} \frac{\partial \ln \left(T n_{i}^{1.6}\right)}{\partial x}\right]^{2}\right\}
$$

We replace $\frac{\partial g}{\partial x}$ according to equation (58) cancel one $\tilde{v}_{T}$, and employ $h=h^{\prime} \frac{\partial \hat{T}}{\partial x}$ :

$$
\begin{gathered}
\left(h^{\prime}+1.833\right)= \\
T_{36} \tilde{v}_{T}\left[-\frac{B_{\phi}}{B_{\theta}}\right]^{2}\left\{\frac{1}{\left[\frac{\partial \hat{T}}{\partial x}\right]} \hat{T} \frac{0.107 q^{2}}{1+\frac{Q^{2}}{S^{2}}} \frac{\partial \ln T}{\partial x} h^{\prime}+\frac{1}{2} \frac{1}{\left[\frac{\partial \hat{T}}{\partial x}\right]^{2}}\left[\frac{B_{\theta}}{B_{\phi}}\right]^{2} g^{2}\right. \\
-\frac{1}{\left[\frac{\partial \hat{T}}{\partial x}\right] 2} g \frac{B_{\theta}}{B_{\phi}}\left(h-\hat{T} \frac{\partial \ln \left(T n_{i}^{2}\right)}{\partial x}\right) \\
\left.+1.9 \frac{1}{\left[\frac{\partial \hat{T}}{\partial x}\right]^{2}}\left[h-0.8 \hat{T} \frac{\partial \ln \left(T n_{i}^{1.6}\right)}{\partial x}\right]^{2}\right\}
\end{gathered}
$$

Using $v_{T}=\tilde{v}_{T} \frac{L_{T}}{L_{\psi}}$ and

$$
\Lambda_{r}=\frac{\nu_{i i}}{\Omega_{i}} \frac{q^{2} R^{2}}{L_{T^{r}}}
$$

we get for $T_{36}\left[\frac{B_{\phi}}{B_{\theta}}\right]^{2}$ successively the relations

$$
\begin{gathered}
T_{36}\left[\frac{B_{\phi}}{B_{\theta}}\right]^{2}=0.36 \frac{6}{50.96} \frac{\nu_{i i}^{2}}{\Omega_{i}^{2}} \frac{1}{\left(1+\frac{Q^{2}}{S^{2}}\right)} q^{2} \frac{1}{\tilde{v}_{T} \frac{L_{T}}{L_{\psi}}} \frac{R^{2}}{L_{\psi}^{2}} \frac{\partial \ln T}{\partial x} \frac{B_{\phi}^{2}}{B_{\theta}^{2}} \\
T_{36}\left[\frac{B_{\phi}}{B_{\theta}}\right]^{2}=0.36 \frac{6}{50.96} \frac{\nu_{i i}^{2}}{\Omega_{i}^{2}} \frac{1}{\left(1+\frac{Q^{2}}{S^{2}}\right)} q^{2} \frac{1}{\tilde{v}_{T} \frac{L_{T}}{L_{\psi} \hat{T}} \hat{T}} \frac{R^{2}}{L_{\psi} L_{T}} \frac{B_{\phi}^{2}}{B_{\theta}^{2}} \\
T_{36}\left[\frac{B_{\phi}}{B_{\theta}}\right]^{2}=0.36 \frac{6}{50.96} \frac{\nu_{i i}^{2}}{\Omega^{2}} \frac{1}{\left(1+\frac{Q^{2}}{S^{2}}\right)} q^{2} \frac{R^{2}}{\tilde{v}_{T} L_{T}^{2}} \frac{B_{\phi}^{2}}{B_{\theta}^{2}} \\
T_{36}\left[\frac{B_{\phi}}{B_{\theta}}\right]^{2}=0.45 \frac{\Lambda_{r}^{2} r^{2}}{q^{2} R^{2}} \frac{1}{\left(1+\frac{Q^{2}}{S^{2}}\right)} \frac{1}{\tilde{v}_{T}} \frac{B_{\phi}^{2}}{B_{\theta}^{2}}
\end{gathered}
$$




$$
T_{36}\left[\frac{B_{\phi}}{B_{\theta}}\right]^{2}=0.45 \frac{\Lambda_{r}^{2}}{\left(1+\frac{Q^{2}}{S^{2}}\right)} \frac{1}{\tilde{v}_{T}}
$$

and finally

$$
T_{36}\left[\frac{B_{\phi}}{B_{\theta}}\right]^{2} \tilde{v}_{T}=0.45 \frac{\Lambda_{r}^{2}}{\left(1+\frac{Q^{2}}{S^{2}}\right)}
$$

With $C_{\eta}=v_{T} \hat{T} T_{36}$ we obtain

$$
T_{36}\left[\frac{B_{\phi}}{B_{\theta}}\right]^{2} \tilde{v}_{T}=\frac{C_{\eta}}{\hat{T}}\left[\frac{B_{\phi}}{B_{\theta}}\right]^{2} \frac{\partial T}{\partial x}=0.45 \frac{\Lambda_{r}^{2}}{\left(1+\frac{Q^{2}}{S^{2}}\right)}
$$

For $h^{\prime}$ we get the equation

$$
\begin{gathered}
\left(h^{\prime}+1.833\right)= \\
0.45 \frac{\Lambda_{r}^{2}}{\left(1+\frac{Q^{2}}{S^{2}}\right)}\left\{\frac{1}{\left[\frac{\partial \hat{T}}{\partial x}\right]} \hat{T} \frac{0.107 q^{2}}{1+\frac{Q^{2}}{S^{2}}} \frac{\partial \ln T}{\partial x} h^{\prime}+\frac{1}{2} \frac{1}{\left[\frac{\partial \hat{T}}{\partial x}\right]^{2}}\left[\frac{B_{\theta}}{B_{\phi}}\right]^{2} g^{2}\right. \\
-\frac{1}{\left[\frac{\partial \hat{T}}{\partial x}\right]^{2}} g \frac{B_{\theta}}{B_{\phi}}\left(h-\hat{T} \frac{\partial \ln \left(T n_{i}^{2}\right)}{\partial x}\right) \\
\left.+1.9 \frac{1}{\left[\frac{\partial \hat{T}}{\partial x}\right]^{2}}\left[h-0.8 \hat{T} \frac{\partial \ln \left(T n_{i}^{1.6}\right)}{\partial x}\right]^{2}\right\}
\end{gathered}
$$

We use (appendix C)

$$
\Lambda_{r}^{2}=\Lambda_{r, i n f}^{2} \hat{T}^{-2 c}\left[\frac{\partial \hat{T}}{\partial x}\right]^{2}
$$

with

$$
2 c=5-\frac{2}{\eta}-2 Z
$$

or

$$
Z=\frac{5}{2}-\frac{1}{\eta}-c
$$


E. g., the following cases are envisaged in chapter (13):

1. $\mathrm{c}=1, \mathrm{Z}=0 \rightarrow \eta=\frac{2}{3}$

2. $\mathrm{c}=1, \eta=\frac{3}{2} \rightarrow \mathrm{Z}=\frac{5}{6}=0.833$

3. $\mathrm{c}=1, \eta=1.6 \rightarrow \mathrm{Z}=0.875$

The choice $\mathrm{c}=1$ is important for the analytical treatment. Using the model profile (51) we have

$$
\begin{gathered}
\frac{\partial \hat{T}}{\partial x}=\frac{\partial(1-\tanh (x))}{\partial x}=-\frac{1}{\cosh ^{2}(x)}= \\
-\left(1-\tanh ^{2}(x)\right)=-(1-\tanh (x))(1+\tanh (x))=-\hat{T}(2-\hat{T})
\end{gathered}
$$

We rewrite equation (63) as

$$
\begin{aligned}
& h^{\prime}+1.833=\frac{0.45 \Lambda}{1+\frac{Q^{2}}{S^{2}}}\left\{\frac{0.107 q^{2}}{1+\frac{Q^{2}}{S^{2}}} h^{\prime}+\frac{1}{2} \frac{1}{\left[\frac{\partial \hat{T}}{\partial x}\right]^{2}}\left(g^{\prime}\right)^{2}\right. \\
& -\frac{1}{\frac{\partial \hat{T}}{\partial x}} g^{\prime}\left(h^{\prime}-\left(1+\frac{2}{\eta}\right)+1.9\left[h^{\prime}-0.8\left(1+\frac{1.6}{\eta}\right]^{2}\right\}\right.
\end{aligned}
$$

Now we assume $h^{\prime}=0$ and get an equation for $\Lambda=\Lambda_{0}$ at the zero of $h^{\prime}$

$$
\begin{gathered}
1.83=\frac{0.45 \Lambda_{0}^{2}}{1+0.26\left[0.625\left(1+2 \eta_{i}^{-1}\right)\right]^{2} \Lambda_{0}^{2}}\left\{0.5 \frac{\left(g_{0}^{\prime}\right)^{2}}{\hat{T}_{0}^{2}\left(2-\hat{T}_{0}\right)^{2}}\right. \\
\left.+\frac{g_{0}^{\prime}}{\hat{T}_{0}\left(2-\hat{T}_{0}\right)}\left(1+2 \eta_{i}^{-1}\right)+1.9\left[0.8\left(1+1.6 \eta_{i}^{-1}\right)\right]^{2}\right\}
\end{gathered}
$$


$T_{0}, g_{0}^{\prime}$ are temperature and the normalized velocity $g^{\prime}$ at the zero of $h^{\prime}$. We approximate the solution by

$$
h^{\prime}=h_{-\infty}^{\prime}\left(1-\frac{\Lambda^{2}}{\Lambda_{0}^{2}}\right)
$$

For small $\Lambda_{r}$ we get the solution at the magnetic axis.

We use the $\mathrm{u}_{\theta}$ - equation and take the limit for $\mathrm{x}=-\infty$. Only the term with $g^{\prime}$ remains

$$
\left(h^{\prime}\right)_{-\infty}=-1.83+\frac{0.45}{\hat{T}^{2 c}} \Lambda_{r}(-\infty)[\hat{T}(2-\hat{T})]^{2} \frac{1}{2}\left(g^{\prime}\right)_{-\infty}^{2} \frac{1}{[\hat{T}(2-\hat{T})]^{2}}
$$

or

$$
\left(h^{\prime}\right)_{-\infty}=-1.83+\frac{0.225}{2^{2 c}} \Lambda_{r}^{2}(-\infty)\left(g^{\prime}\right)_{-\infty}^{2}
$$

We use the equation

$$
\frac{Q}{S}=0.5\left(h^{\prime}-0.625\left(1+\frac{2}{\eta}\right)\right) \Lambda_{r}
$$

and combine it with the Ansatz for $h^{\prime}$

$$
\frac{Q}{S}=0.5\left(\left(h^{\prime}\right)_{-\infty}\left(1-\frac{\Lambda_{r}^{2}}{\Lambda_{0}^{2}}\right)-0.625\left(1+\frac{2}{\eta}\right)\right) \Lambda_{r}
$$

From the relation

$$
f^{2}=\frac{\Lambda_{r}^{2}}{\Lambda_{0}^{2}}
$$

we get

$$
f=\frac{\Lambda_{r, i n f}}{\Lambda_{0}} \frac{2-\hat{T}}{\hat{T}^{c-1}}
$$

At the boundary we obtain

$$
1=\frac{\Lambda_{r, \text { inf }}}{\Lambda_{0}} \frac{2-\hat{T}_{0}}{\hat{T}_{0}^{c-1}}
$$


We get

$$
\frac{Q}{S}=0.5\left(\left(h^{\prime}\right)_{-\infty}-0.625\left(1+\frac{2}{\eta}\right)-\left(h^{\prime}\right)_{-\infty} f^{2}\right) \Lambda_{r}
$$

or - if we replace $\Lambda_{r}$ -

$$
\frac{Q}{S}=0.5\left(\left(h^{\prime}\right)_{-\infty}-0.625\left(1+\frac{2}{\eta}\right)-\left(h^{\prime}\right)_{-\infty} f^{2}\right) \Lambda_{0} f
$$

We discuss

$$
F=\frac{2-\hat{T}}{\hat{T}^{c-1}}
$$

as a function of $\hat{T}$. F has an extremum for

$$
\hat{T}=2 \frac{c-1}{c-2}
$$

We investigate $\frac{Q^{2}}{S^{2}}$ as function of $f^{2}$. It has a maximum for

$$
f^{2}=f_{\max }^{2}=\frac{1}{3}\left[1-\frac{0.625\left(1+\frac{2}{\eta}\right)}{\left(h^{\prime}\right)_{-\infty}}\right]
$$

and a minimum for

$$
f^{2}=f_{\min }^{2}=1-\frac{0.625\left(1+\frac{2}{\eta}\right)}{\left(h^{\prime}\right)_{-\infty}}
$$

This value is larger than unity and outside the range of interest.

The maximum value is

$$
\frac{Q^{2}}{S^{2}}=0.26 \Lambda_{0}^{2}\left[\left(h^{\prime}\right)_{-\infty}\right]^{2} \frac{4}{27}\left[1-\frac{0.625\left(1+\frac{2}{\eta}\right)}{\left(h^{\prime}\right)_{+\infty}}\right]^{3}
$$

We approximate $\frac{Q^{2}}{S^{2}}$ by a parabola which interpolates between $\mathrm{f}=0, \mathrm{f}=f_{m}$ and $\mathrm{f}=1$. We get

$$
\frac{Q^{2}}{S^{2}}=0.26 \Lambda_{0}^{2}\left[\left(h^{\prime}\right)_{-\infty}\right]^{2}\left[f_{m}^{2}\left(9 f_{m}^{2}-1\right)-\left(5 f_{m}^{2}-1\right) f^{2}\right] f^{2}
$$


The toroidal velocity is then given by

$$
g^{\prime}(\widehat{T})=g^{\prime}\left(\widehat{T}_{0}\right)+0.107 q^{2}\left(h^{\prime}\right)_{-\infty} \int_{\widehat{T}_{0}}^{\widehat{T}} \frac{\left(1-f^{2}\right)(2-\widehat{T}) d \widehat{T}}{1+\alpha f^{2}+\beta f^{4}}
$$

$\alpha$ and $\beta$ are given by

$$
\alpha=0.26 \Lambda_{0}^{2}\left(h^{\prime}\right)_{-\infty}^{2} f_{m}^{2}\left(9 f_{m}-1\right)
$$

and

$$
\beta=-0.26 \Lambda_{0}^{2} h_{-\infty}^{\prime 2} f_{m}^{2}\left(5 f_{m}-1\right)
$$

One can factorize $1+\alpha f^{2}+\beta f^{4}$ and gets

$$
1+\alpha f^{2}+\beta f^{4}=\beta\left(f^{2}-a\right)\left(f^{2}-b\right)
$$

where

$$
\left(\begin{array}{l}
a \\
b
\end{array}\right)=\frac{-\alpha \pm \sqrt{\alpha^{2}-4 \beta}}{2 \beta}
$$

In the expression

$$
f=\frac{\Lambda_{r, i n f}}{\Lambda_{0}} \frac{2-\hat{T}}{\hat{T}^{c-1}}
$$

we put $\hat{T}=\hat{T}_{0}$ and get

$$
1=\frac{\Lambda_{r, \text { inf }}}{\Lambda_{0}} \frac{2-\hat{T}_{0}}{\hat{T}_{0}^{c-1}}
$$

or

$$
\begin{gathered}
\frac{\Lambda_{r, i n f}}{\Lambda_{0}}=\frac{\hat{T}_{0}^{c-1}}{2-\hat{T}_{0}} \\
\Lambda_{0}=\Lambda_{r, i n f} \frac{2-\hat{T}_{0}}{\hat{T}_{0}^{c-1}}
\end{gathered}
$$


We consider $\mathrm{c}=1$ implying

$$
f=\frac{\Lambda_{r, i n f}}{\Lambda_{0}}(2-\hat{T})
$$

because in this case a simple analytical integration is possible.

This integration yields the jump

$u_{\phi}(\hat{T}=2)-u_{\phi}\left(\hat{T}_{0}\right)=-\frac{0.107 q^{2}\left(h^{\prime}\right)_{-\infty}}{2 \sqrt{\alpha^{2}-4 \beta}} \frac{\Lambda_{0}}{\Lambda_{r, \text { inf }}} \ln \left[\left[\frac{-a}{1-a}\right]^{1-a}\left[\frac{-b}{b-1}\right]^{b-1}\right]$

The radial dependance is given by

$\left.u_{\phi}(\hat{T})-u_{\phi}\left(\hat{T}_{0}\right)=-\frac{0.107 q^{2}\left(h^{\prime}\right)_{-\infty}}{2 \sqrt{\alpha^{2}-4 \beta}} \frac{\Lambda_{0}}{\Lambda_{r, i n f}} \ln \left[f^{2}-a\right]^{1-a}\left[b-f^{2}\right]^{b-1}\right]$

We assume

$$
\eta=1.6
$$

and (neglecting the small term $\left.\frac{0.225}{2^{2 c}} \Lambda_{r}^{2}(-\infty)\left(g^{\prime}\right)_{-\infty}^{2}\right)$ approximately)

$$
\left(h^{\prime}\right)_{-\infty}=-1.83
$$

and use the boudary value

$$
\left(h^{\prime}\right)_{r_{s}}=0
$$

to compute $\Lambda_{0}^{2}$. We get the equation

$$
\begin{gathered}
1.83=\frac{0.45 \Lambda_{0}^{2}}{1+0.26\left[0.625\left(1+2 \eta_{i}^{-1}\right)\right]^{2} \Lambda_{0}^{2}}\left\{0.5 \frac{u_{\phi, 0}^{2}}{\hat{T}_{0}^{2}\left(2-\hat{T}_{0}\right)^{2}}\right. \\
\left.+\frac{u_{\phi, 0}}{\hat{T}_{0}\left(2-\hat{T}_{0}\right)}\left(1+2 \eta_{i}^{-1}\right)+1.9\left[0.8\left(1+1.6 \eta_{i}^{-1}\right)\right]^{2}\right\}
\end{gathered}
$$


In addition we assume $u_{\phi, 0}=0$ (concerning the boundary values also see chapter 6 ) and get

$$
\begin{aligned}
1.83= & \frac{0.45 \Lambda_{0}^{2}}{1+0.26\left[0.625\left(1+2 \eta_{i}^{-1}\right)\right]^{2} \Lambda_{0}^{2}}\{ \\
& \left.+1.9\left[0.8\left(1+1.6 \eta_{i}^{-1}\right)\right]^{2}\right\}
\end{aligned}
$$

or

$$
\left.1.83+1.830 .26\left[0.625\left(1+2 \eta_{i}^{-1}\right)\right]^{2} \Lambda_{0}^{2}\right)=0.45 \Lambda_{0}^{2}\left\{+1.9\left[0.8\left(1+1.6 \eta_{i}^{-1}\right)\right]^{2}\right\}
$$

Therefore

$\Lambda_{0}^{2}\left(1.830 .26\left[0.625\left(1+2 \eta_{i}^{-1}\right)\right]^{2}-0.451 .9\left[0.8\left(1+1.6 \eta_{i}^{-1}\right)\right]^{2}\right)=-1.83$

or

$$
\Lambda_{0}^{2}=\frac{-1.83}{\left(1.830 .26\left[0.625\left(1+2 \eta_{i}^{-1}\right)\right]^{2}-0.451 .9\left[0.8\left(1+1.6 \eta_{i}^{-1}\right)\right]^{2}\right)}
$$

and finally

$$
\Lambda_{0}^{2}=1.47
$$

$g^{\prime}$ and $h^{\prime}$ allow to compute the radial electric field which is given by

$$
E_{r}=B_{\theta} u_{\phi}-B_{\phi} u_{\theta}+\frac{T_{i}}{e_{i}} \frac{\partial P_{i}}{\partial r}
$$

This can be rewritten as

$$
\frac{E_{r}}{B_{\phi}}=\frac{B_{\theta}}{B_{\phi}} u_{\phi}-u_{\theta}+\frac{T}{e B_{\phi} L_{\psi}} \frac{1}{n T}\left[n \frac{\partial T}{\partial x}+T \frac{\partial n}{\partial x}\right]
$$

Introducing the ratio $\eta$ entails

$$
\frac{E_{r}}{B_{\phi}}=\frac{B_{\theta}}{B_{\phi}} u_{\phi}-u_{\theta}+\frac{T_{i}}{e_{i} B_{\phi} L_{\psi}} \frac{1}{\hat{T}} \frac{\partial \hat{T}}{\partial x}\left[1+\frac{1}{\eta}\right]
$$


or with the help of the velocity $v_{T}$

$$
\frac{E_{r}}{B_{\phi}}=v_{T}\left\{\frac{B_{\theta}}{B_{\phi}} g-h+\frac{\partial \hat{T}}{\partial x}\left[1+\frac{1}{\eta}\right]\right\}
$$

We introduce $g^{\prime}$ and $h^{\prime}$ and get

$$
\frac{E_{r}}{B_{\phi}}=v_{T}\left\{g^{\prime}+\frac{\partial \hat{T}}{\partial x}\left[-h^{\prime}+1+\frac{1}{\eta}\right]\right\}
$$

The radial electric field is then

$$
E_{r}=\frac{T_{i n f}}{e_{i} L_{\psi}}\left[g^{\prime}+\left(-h^{\prime}+1+\frac{1}{\eta}\right) \frac{\partial \hat{T}}{\partial x}\right]
$$




\section{Numerical solution method}

The second order equation is replaced by two first order equations. The 1st equation is that for $\frac{\partial g}{\partial x}$ which can be written as

$$
\frac{\partial g}{\partial x}=G+\frac{0.107 q^{2}}{1+\frac{Q^{2}}{S^{2}}} \frac{\partial \ln T}{\partial x} \frac{B_{\phi}}{B_{\theta}} h
$$

The 2nd equation is that for $G$ which is related to the first derivative of $g$ by

$$
G=\frac{\partial g}{\partial x}-\frac{0.107 q^{2}}{1+\frac{Q^{2}}{S^{2}}} \frac{\partial \ln T}{\partial x} \frac{B_{\phi}}{B_{\theta}} h
$$

Thus the 2nd equation looks like

$$
\begin{aligned}
& \frac{\partial}{\partial x} G=\frac{m_{i} n_{i n f} L_{\psi}^{2}}{\eta_{2, i n f} \hat{\eta}_{2}}\left[\hat{n} \nu_{c x} g-\frac{\hat{n} \dot{M}_{\phi, i}}{v_{T}}+\right. \\
& \left.\frac{J_{r} B_{\theta}}{m_{i} v_{T} n_{i n f}}-\frac{\eta_{2, i n f}}{m_{i} n_{i n f} L_{\psi}^{2}} \hat{\eta}_{2} G \frac{\partial}{\partial x} \ln \left(\eta_{2}\right)\right]
\end{aligned}
$$

or

$$
\begin{gathered}
\frac{\partial}{\partial x} G=\frac{1}{\eta^{\prime} \hat{\eta}_{2}}\left[\hat{n}+\nu_{c x} g-\frac{\hat{n} \dot{M}_{\phi, i}}{v_{T}}+\right. \\
\left.\frac{J_{r} B_{\theta}}{m_{i} v_{T} n_{i n f}}-\eta^{\prime} \hat{\eta}_{2} G \frac{\partial}{\partial x} \ln \left(\eta_{2}\right)\right]
\end{gathered}
$$

In addition we have the equation for the poloidal rotation which can be written as a nonlinear equation for $h$

$$
\begin{gathered}
\left(h+1.833 \frac{\partial \hat{T}}{\partial x}\right) \hat{T}=T_{45}\left[\frac{B_{\phi}}{B_{\theta}} \hat{T} \frac{\partial g}{\partial x}+\frac{1}{2} g^{2}\right. \\
-g \frac{B_{\phi}}{B_{\theta}}\left(h-\hat{T} \frac{\partial \ln \left(T n_{i}^{2}\right)}{\partial x}\right)
\end{gathered}
$$




$$
\left.+1.9 \frac{B_{\phi}^{2}}{B_{\theta}^{2}}\left[h-0.8 \hat{T} \frac{\partial \ln \left(T n_{i}^{1.6}\right)}{\partial x}\right]^{2}\right]
$$

For a given temperature and density profile and the radial dependence of the normalized toroidal velocity $g$ this equation is solved for $h$ by means of the solver ZEROIN [28] for transcendental equations. 


\section{Friction due to recycled neutrals}

The charge exchange frequency of a neutral gas with the density $n_{0} \frac{1}{m^{3}}$ is given by

$$
\nu_{c x}=4.7 \quad 10^{-14} \frac{m^{3}}{\sec } n_{0} \frac{1}{m^{3}}
$$

We multiply with the characteristic time (8) and get as charge exchange term in equation (67)

$$
T_{C X}=\frac{\hat{n}}{\hat{\eta}_{2}} t_{c} \nu_{c x} g=\hat{T}^{0.5-Z_{t}} t_{c} \nu_{c x} g
$$

We assume that the neutral gas density decays as

$$
n_{0}=N_{0} \exp \left(\frac{L_{\psi}}{L_{\text {neu }}} x\right)
$$

$N_{0}$ is the neutral gas density at the inflection point.

In the case of the Alcator [30] we have $t_{c}=3.310^{-3} \mathrm{sec}, N_{0}=$ $510^{14}$ and one gets

$$
T_{c x}\left(r=r_{i n f}\right)=4.710^{-14} 510^{14} 3.310^{-3}=8.0810^{-2}
$$

This has an influence on the step $g-g_{0}$ already. 


\section{Neutral injection}

The injected current $I_{b}$ in Amp gives rise to an increase of the mean density by

$$
\dot{N}_{0}=\frac{I_{b}[A]}{e_{0}}
$$

$e_{0}$ is the elementary charge. Resorting to the injected power $\mathrm{P}$ and the beam energy $E_{0}$ we get

$$
\dot{N}_{0}=\frac{P_{M W}}{E_{0, k e V}} \frac{10^{3}}{e_{0}}
$$

or

$$
\dot{N}_{0}=0.62410^{22} \frac{P_{M W}}{E_{0, k e V}}
$$

At large energies the rate coefficient $\langle\sigma v\rangle_{c x}$ saturates at

$$
<\sigma v>_{c x}=1.510^{-7} \frac{\mathrm{cm}^{3}}{\mathrm{sec}}
$$

The charge exchange frequency is

$$
<\sigma v>_{c x} \dot{N}_{0}=1.510^{-13} \frac{m^{3}}{s e c} 0.62410^{22} \frac{P_{M W}}{E_{0, k e V}}
$$

or

$$
<\sigma v>_{c x} \dot{N}_{0}=0.9410^{9} \frac{m^{3}}{\sec } \frac{P_{M W}}{E_{0, k e V}}
$$

Acounting for the geometry of a pencil beam and of a circular tokamak plasma [24] we get for the deposited momentum

$$
\dot{M}_{\|, i}=\frac{\dot{N}_{0}<\sigma v>_{c x}}{\sqrt{2 \epsilon}(2 \pi)^{2} r R}
$$


This can be rewritten as

$$
\dot{M}_{\|, i}=0.9410^{7} \frac{-1}{r^{\frac{3}{2}} R^{\frac{1}{2}}} \frac{P_{M W}}{E_{0, k e V}} \frac{100}{\sqrt{2} 4 \pi^{2}}
$$

or as

$$
\dot{M}_{\|, i}=0.9410^{7} \frac{-1}{r^{\frac{3}{2}} R^{\frac{1}{2}}} \frac{P_{M W}}{E_{0, k e V}} 1.79
$$

Finally we have

$$
\dot{M}_{\|, i}=1.6810^{7} \frac{-1}{r^{\frac{3}{2}} R^{\frac{1}{2}}} \frac{P_{M W}}{E_{0, k e V}}
$$




\section{Results}

The equations for $u_{\phi}$ and $u_{\theta}$ are solved numerically (1) to benchmark with a simplified analytical theory for $\Lambda_{1}=0$ and vanishing neutral gas density and (2) to compare with the theory accounting for finite $\Lambda_{1}$ effects and (3) to explore the parameter space in regions where the analytical theory is not valid, in particular in the case where the neutral gas density larger than $10^{17} \mathrm{~m}^{-3}$ and/or neutral injection is important.

The method resorts to an ODE - solver for the classical momentum balance which is combined with a solver for transcendental equations yielding $v_{\Theta}$.

Several integration procedures such as the Adams - Pece -, Burlish - Stoer -, or the fifth order Cash - Carp - Runge - Kutta method were applied showing that the results are almost independent from the special numerical method.

\subsection{Comparison with the simplified analytical theory of section 8 and the halfanalytical theory of sec- tion 7}

. For the case $T_{i n f}=165 \mathrm{eV}, L_{\psi}=0.76 \mathrm{~cm}, r_{i n f}=20.8 \mathrm{~cm}, r_{s}$

$=21.5 \mathrm{~cm}$ (Fig. 1), we get the jump $\Delta u_{\phi}=u_{\phi}-u_{\phi_{0}}=140 \frac{\mathrm{km}}{\mathrm{sec}}$, the analytical and the numerical solutions agree exactly (Fig. $2)$. This jump is much larger than the measured one $\left(\Delta u_{\phi} \approx\right.$ $35 \frac{\mathrm{km}}{\mathrm{sec}}$ ) because the approximation $\frac{Q^{2}}{\mathrm{~S}^{2}}=0$ was made, i. e. in the numerical and the analytical approach $\Lambda_{1}=C_{\eta}=0$ was assumed.

The halfanalytical treatment in chapter (7) resorts to the neoclassical solution (48). One gets exact agreement with the ODE 
- integration (Fig. 3) if in the numerical method the solution of equation (24) is replaced by the neoclassical value $\left(C_{\eta}=0\right)$. The corresponding jump of the approximate solution $\Delta u_{\phi}=43$ $\frac{\mathrm{km}}{\mathrm{sec}}$ is now close to measured one.

Since the neoclassical approximation of $u_{\theta}$ is rather accurate the halfanalytical solution (circles) agrees well with the exact solution (triangles). In Fig. 4 both solution are compared. We see that the 'neoclassical jump' is around 10\% larger than the corresponding jump of the exact numerical solution $\Delta u_{\phi}=35$ $\frac{\mathrm{km}}{\mathrm{sec}}$ which is now close to measured one.

\subsection{Comparison with the approximate analytical the- ory of section 9, accounting for finite $\Lambda_{1}$}

The input data are those of ALCATOR C-MOD [30] (partly used already before): $T_{\text {inf }}=165 \mathrm{eV}, n_{i}=1.8710^{20} \mathrm{~m}^{-3}, L_{\psi}=$ $0.76 \mathrm{~cm}, r_{\text {inf }}=20.8 \mathrm{~cm}, r_{s}=21.5 \mathrm{~cm}, \epsilon=1.6, Z_{\text {eff } f_{\text {inf }}}=1.57, \mathrm{Z}$ $=0.875, \eta=1.6, \mathrm{R}=67 \mathrm{~cm}, B_{\theta}=0.625 \mathrm{~T}$ and $B_{\phi}=5.2 \mathrm{~T}$. Here $Z_{e f f_{\text {inf }}}$ and $\mathrm{Z}$ are defined by $Z_{\text {eff }}(r)=Z_{\text {eff }} \hat{T}^{Z}$, i. e. $Z_{\text {eff } f_{\text {inf }}}$ is $Z_{e f f}(r)$ at the inflection point and $\mathrm{Z}$ is a constant. We proceed with the comparison of numerical and analytical solutions in the case of the exact expression for $\Lambda$. The jumps $\Delta u_{\phi}$ and the radial dependence of $u_{\phi}$ according to the numerical integration (triangles) and according to the analytical theory (quadrangles) agree well (Fig. 5). The deviation of the maxima (jumps) is $\approx 8 \%$. Both jumps, $\Delta u_{\phi}=34 \frac{\mathrm{km}}{\mathrm{sec}}$ (numerical) and $\Delta u_{\phi}=37$ $\frac{\mathrm{km}}{\mathrm{sec}}$ (analytical), agree approximately with the experimental one $\left(\approx 35 \frac{\mathrm{km}}{\mathrm{sec}}\right)$ [30]. The deviations of the maxima of $u_{\theta}$ and of the minima of $E_{r}$ according to the numerical integration and 
the analytical theory are less than $\approx 8 \%$ and $10 \%$, respectively (Fig. 6 and Fig. 7). The radial dependence shown in Fig. 7 is similar to that reported at DIII - D.

\subsection{General radial profiles of the plasma parameters}

In Fig. 8 the profile

$$
\hat{T}=\hat{T}_{0}+\left(1-\hat{T}_{0}\right) \exp \left(-\frac{x}{1-\hat{T}_{0}}\right)
$$

for $\mathrm{x}<0$ and

$$
\hat{T}=\exp [-x]
$$

for $\mathrm{x}>0$, discussed in appendix $\mathrm{A}$ in more detail, is shown. This profile is continuous, has a contiuous derivative, but in contrast to the profile (51) the second derivative is discontinuous. In Fig. 9 the normalized velocity $\mathrm{g}$ is shown in the range $[\mathrm{x}=-$ $12, \ldots, \mathrm{x}=1.4]$. As in the solutions before the toroidal velocity increases within the distance $L_{\psi}$ to roughly the same value as before in Fig. 5. The poloidal velocity (Fig. 10) has a sharp maximum of $10 \frac{\mathrm{km}}{\mathrm{sec}}$. It becomes zero at $\mathrm{r}=22 \mathrm{~cm}$. Analogously the electric field (Fig. 11) has a sharp local minimum.

We now generalize the profile (51) to

$$
T=T_{\text {inf }}\left\{(1-\tanh (x))+\left(\frac{T_{0}}{T_{\text {inf }}}-2\right)\left(1-\left[\frac{r}{r_{\text {inf }}}\right]^{2}\right)^{2}\right\}
$$

$T_{0}$ is the temperature at the plasma center (Fig. 12). This profile has been measured at ALCATOR C - MOD [30]. In Fig. $13 \mathrm{~g}$ is shown in the same range as before in Fig. 5. The major part of the step in $u_{\phi}$ is located in the boundary region. $u_{\phi}$ increases there to around $32 \frac{\mathrm{km}}{\mathrm{sec}}$. In the central part of the plasma the 
increase is around $10 \frac{\mathrm{km}}{\mathrm{sec}}$. ( $T_{0}=1020 \mathrm{eV}$ was assumed.) The poloidal velocity has a dominant peak in the boundary region (Fig. 14), and the electric field is somewhat modified in the central region (Fig. 15). However, one has to keep in mind the restricted validity of these calculation including thethe central core plasma, because there another collisionality regime may be entered where modifications of the present theory are needed.

\subsection{Influence of the neutral gas}

It turns out that the solution is very sensitive with respect to the neutral gas density. This may indicate the the neutral gas plays a decisive role in the $\mathrm{L}-\mathrm{H}$ transition. In fact, the neutral gas physics in limiter and divertor devices are quite different. In Fig. 16 the influence of the neutral gas is investigated. Even the very low density of $N_{0}=5 \cdot 10^{14} / \mathrm{m}^{3}$ an effect on the step can be seen which is reduced in size by $20 \%$ and gets a pedestal at the outside. In the poloidal rotation velocity and the radial electric field the influence of such a small neutral gas density can be neglected.

If the neutral density $N_{0}$ is increased to $N_{0}=5 \cdot 10^{15} / \mathrm{m}^{3}$ the step in $\mathrm{g}$ is reduced to about $20 \%$, i. e. almost removed (Fig. 16). Therefore the system may not be able to transit into the $\mathrm{H}$ - mode (Fig. 10).

Since not all charge exchange neutrals leave the plasma and contribute to the momentum loss (as it is assumed in equation [15]), the aforementioned neutral gas densities are probably underestimated by a factor 2 - 3. However, the inclusion of a more detailed neutral gas model would be beyond the scope of this 
report.

\section{Conclusions}

The good agreement between the numerical and the analytical model shows that both models are consistent and the comparison with the experimental data should be reasonable. In fact, this comparison shows that in (ohmic discharges of) the ALCATOR - device neoclassical physics prevail during the H - mode. Surprising is the strong influence of the neutral gas. A very small density $n_{0}=510^{15} \mathrm{~m}^{-3}$ destroys the step already.

Preliminary calculations accounting for neutral beam injection indicate that in this case the neoclassical viscosity must be corrected by an anomalous contribution. 


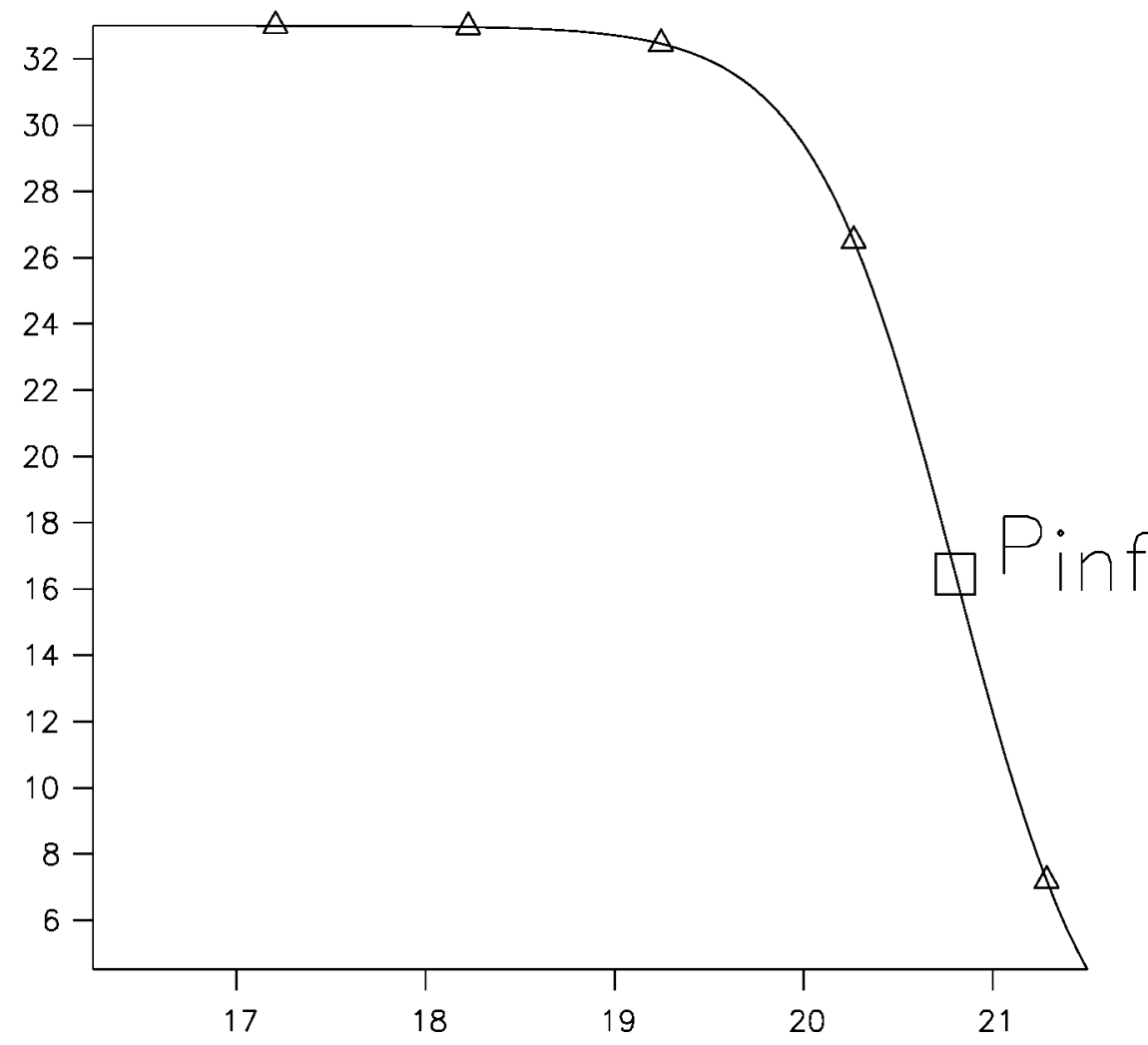

Figure 1: Ion temperature $\mathrm{T}[10 \mathrm{eV}]$. The inflection point of the temperature profile, $P_{\text {inf }}$, is the locus of vanishing curvature. The decaylength there and the maximum at $\mathrm{r}=0(165 \mathrm{eV})$ are adjusted to ALCATOR C-MOD data. 


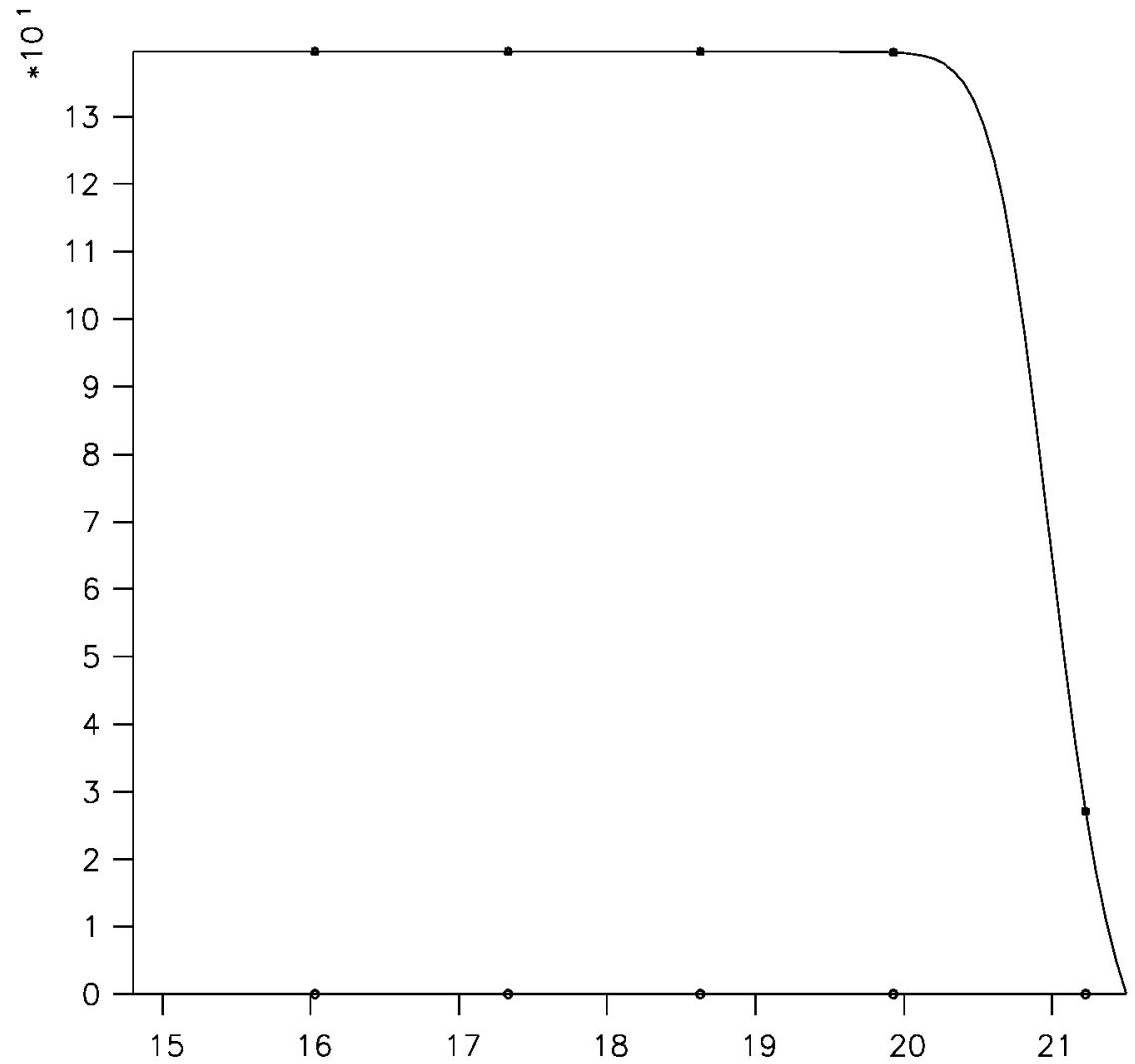

Figure 2: Toroidal velocity $U_{\phi}[\mathrm{km} / \mathrm{sec}]$. Due to the assumptions $\frac{Q}{S}=0$ and $C_{\eta}=0$ the analytical and the numerical solutions agree exactly. By the same reason the jump of the toroidal velocity $\Delta u_{\phi}=u_{\phi}-u_{\phi_{0}}=140 \frac{\mathrm{km}}{\mathrm{sec}}$ is much too large. 


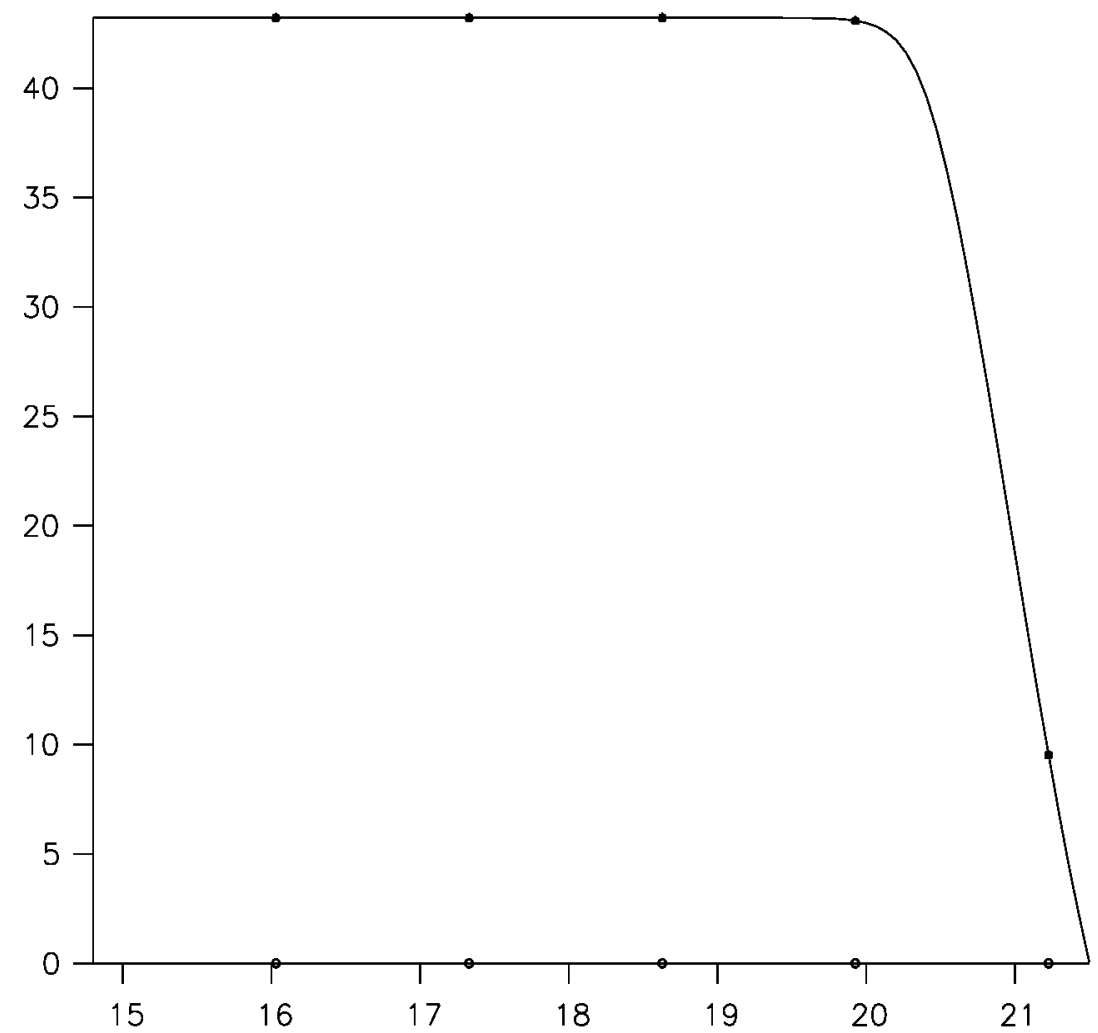

Figure 3: Toroidal velocity $U_{\phi}[\mathrm{km} / \mathrm{sec}]$. The halfanalytical treatment in chapter (7) resorts to the neoclassical solution (48). One gets exact agreement with the ODE - integration if the solution of equation (24) is replaced by the neoclassical value $\left(C_{\eta}=0\right)$. The corresponding jump of the approximate solution $\Delta u_{\phi}=43 \frac{\mathrm{km}}{\mathrm{sec}}$ is now close to measured one. 


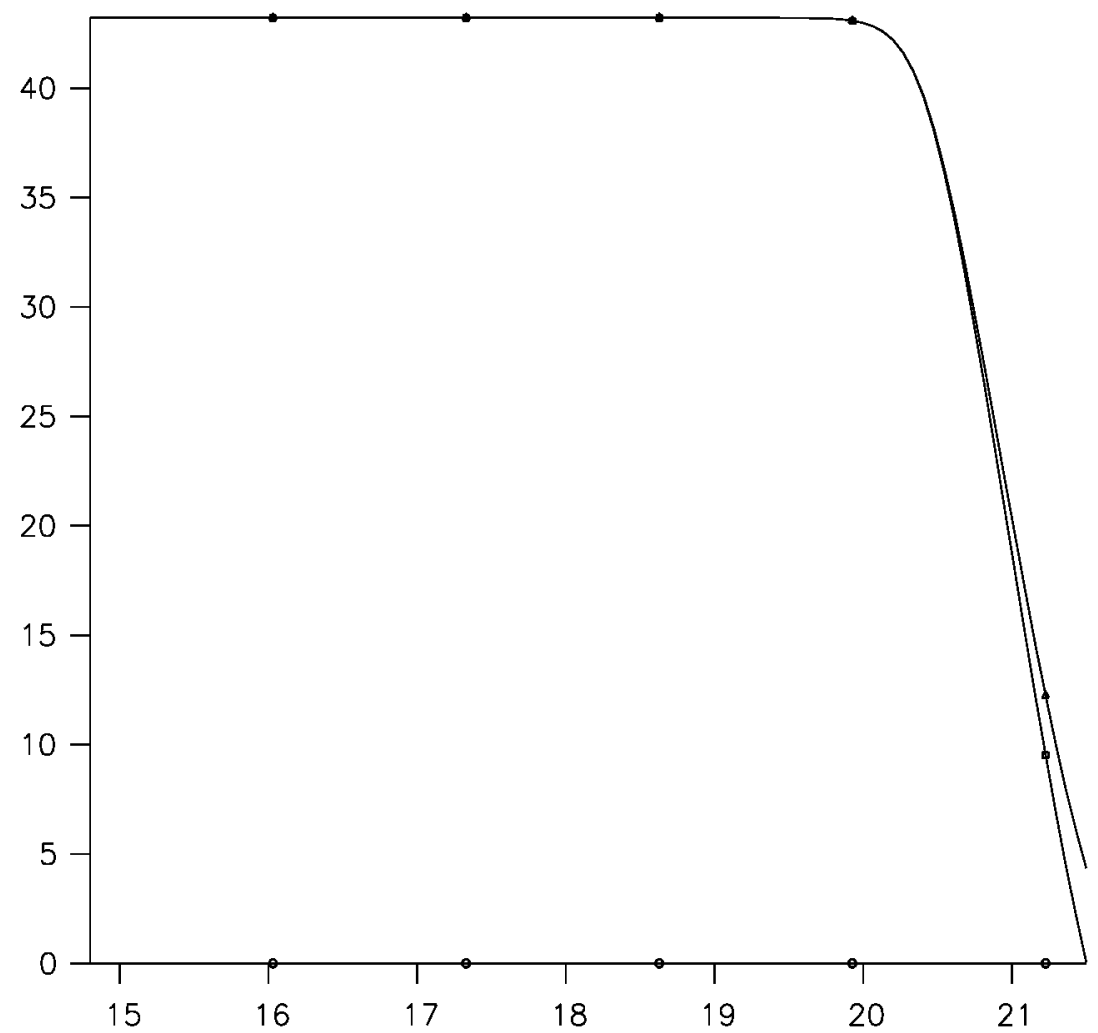

Figure 4: Toroidal velocity $U_{\phi}[\mathrm{km} / \mathrm{sec}]$. One gets approximate agreement of the halfanalytical solutions (triangles) with the exact ODE - integration. As in Fig. 3 the halfanalytical solution is obtained by replacing $u_{\theta}$ by the neoclassical value $\left(C_{\eta}=0\right)$. The corresponding jump of the approximate solution $\Delta u_{\phi}=43 \frac{\mathrm{km}}{\mathrm{sec}}$ is now close to measured one. 


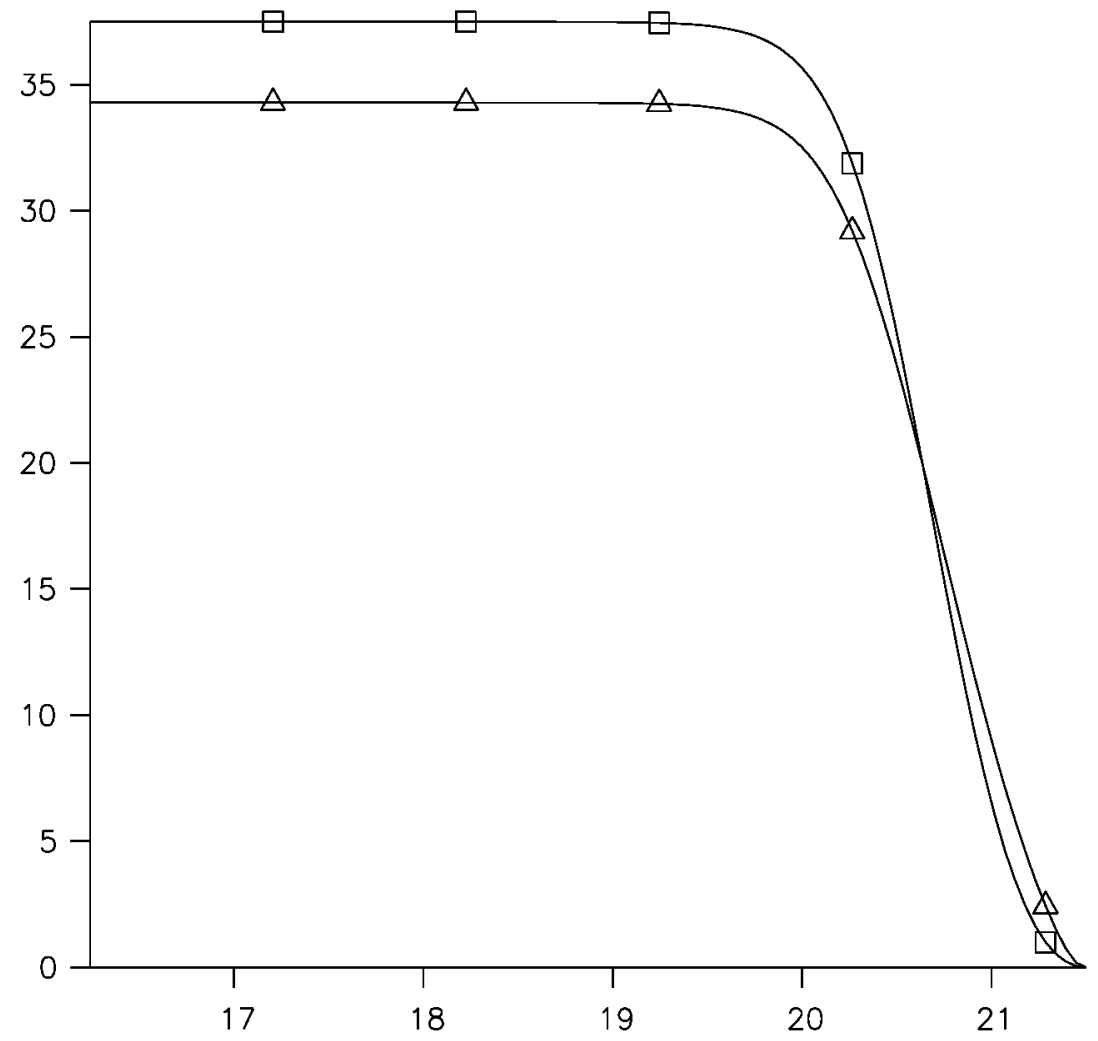

Figure 5: Toroidal velocity $U_{\phi}[\mathrm{km} / \mathrm{sec}]$. The jumps $\Delta u_{\phi}$ and the radial dependence of $u_{\phi}$ according to the numerical integration (triangles) and according to the analytical theory (quadrangles) of chapter (9) agree well. The deviation of the maxima (jumps) is $\approx 8 \%$. Both jumps agree approximately with the experimental one $\left(\approx 35 \frac{\mathrm{km}}{\mathrm{sec}}\right)[30]$. 


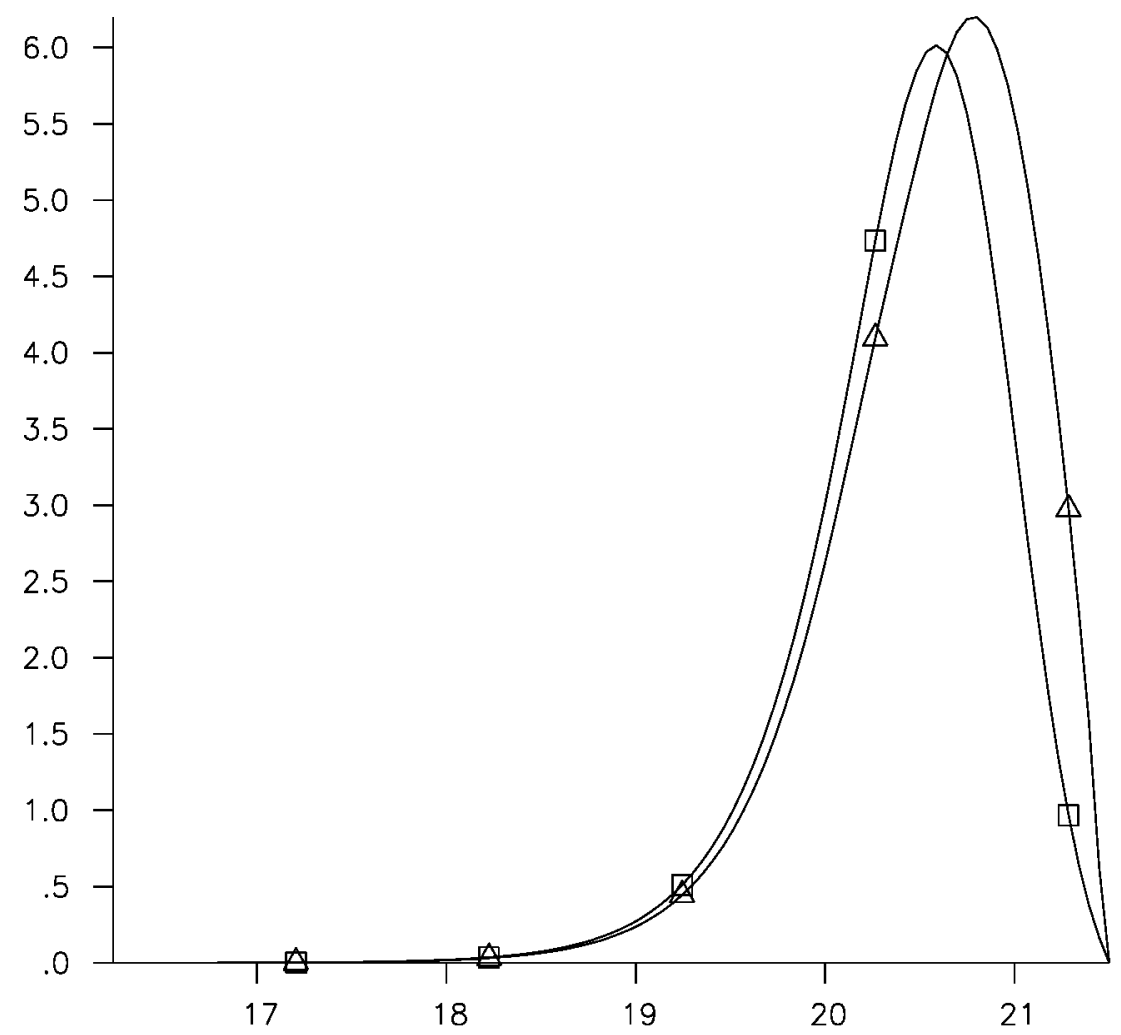

Figure 6: Poloidal velocity $U_{\theta}[\mathrm{km} / \mathrm{sec}]$. The deviations of the maxima of $u_{\theta}$ according to the numerical integration and the analytical theory are less than $\approx 8 \%$. 


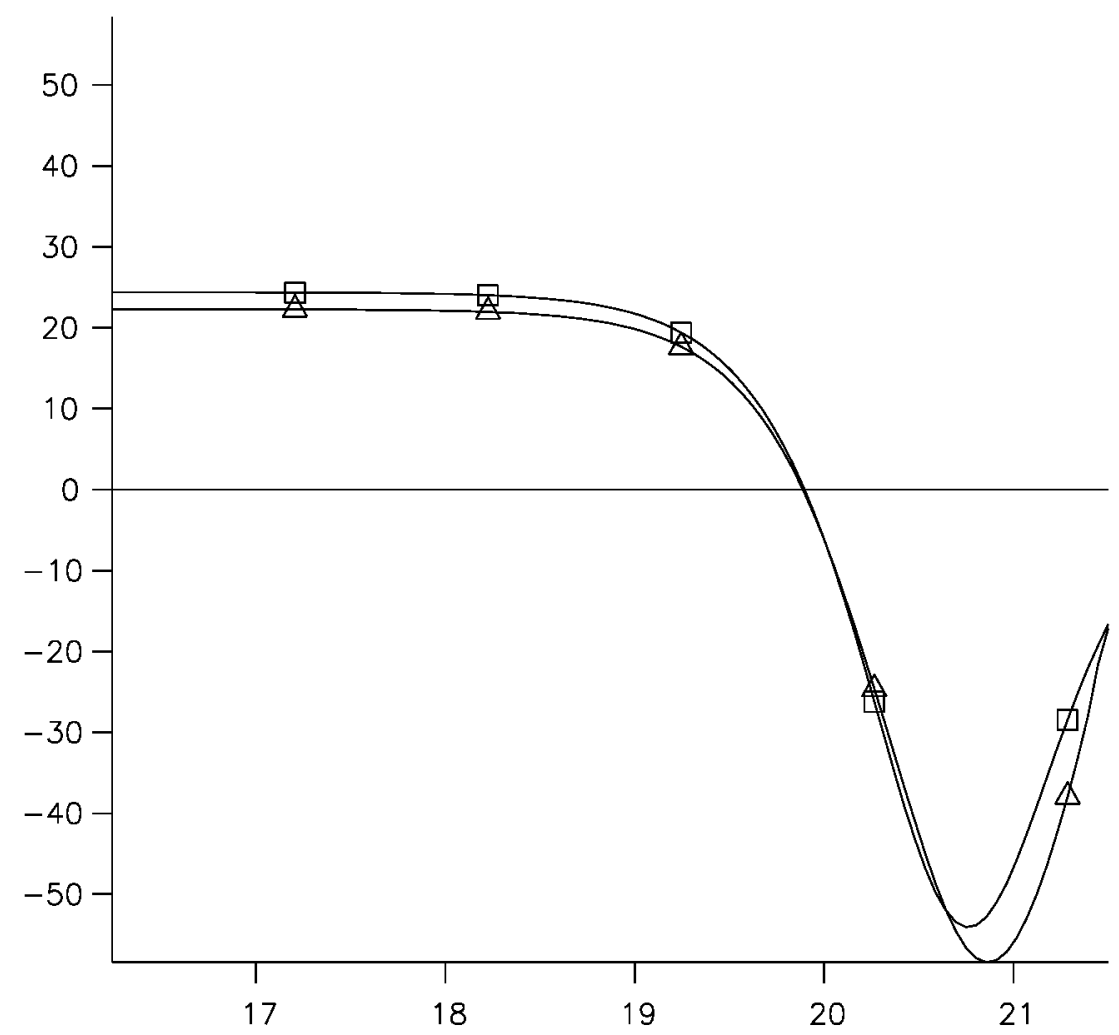

Figure 7: Radial electric field $E_{r}[\mathrm{kV} / \mathrm{m}]$. The deviations of minima of $E_{r}$ according to the numerical integration and the analytical theory are less than $\approx 10 \%$. The radial dependence is similar to that reported at DIII - D. 


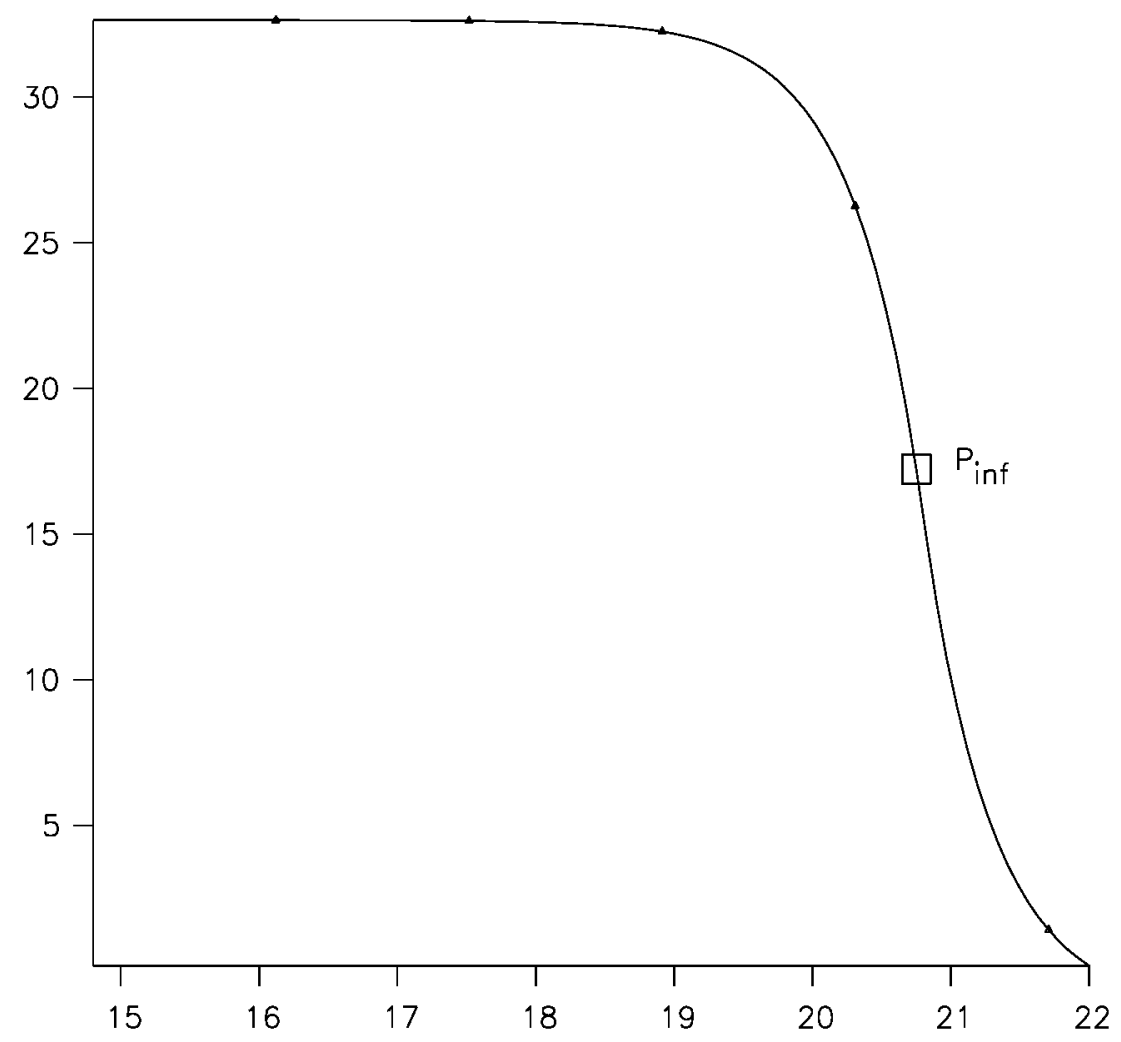

Figure 8: Ion temperature $\mathrm{T}[10 \mathrm{eV}]$. The temperature profile in the appendix (A) was chosen to demonstrate the effect of different profile shapes. It has an inflection point at $r=r_{\text {inf }}$. As in Fig. 1 the central value is 2 times larger than the value at the inflection point, so that similar results can be expected as Figs. 6 and 7. 


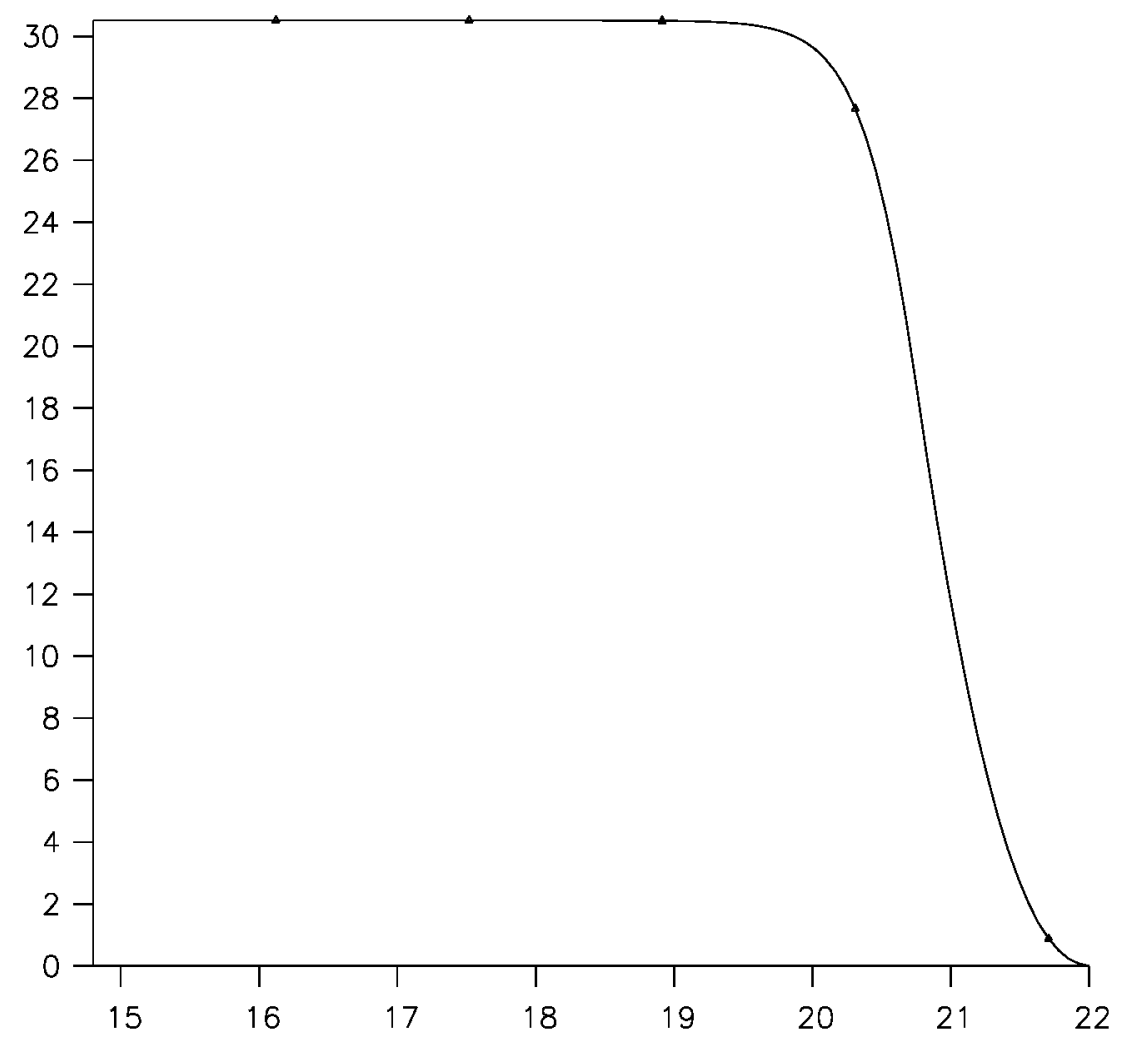

Figure 9: Toroidal velocity $U_{\phi}[\mathrm{km} / \mathrm{sec}]$. The total jump in $\mathrm{g}$ is three times larger than in Fig. 5. 


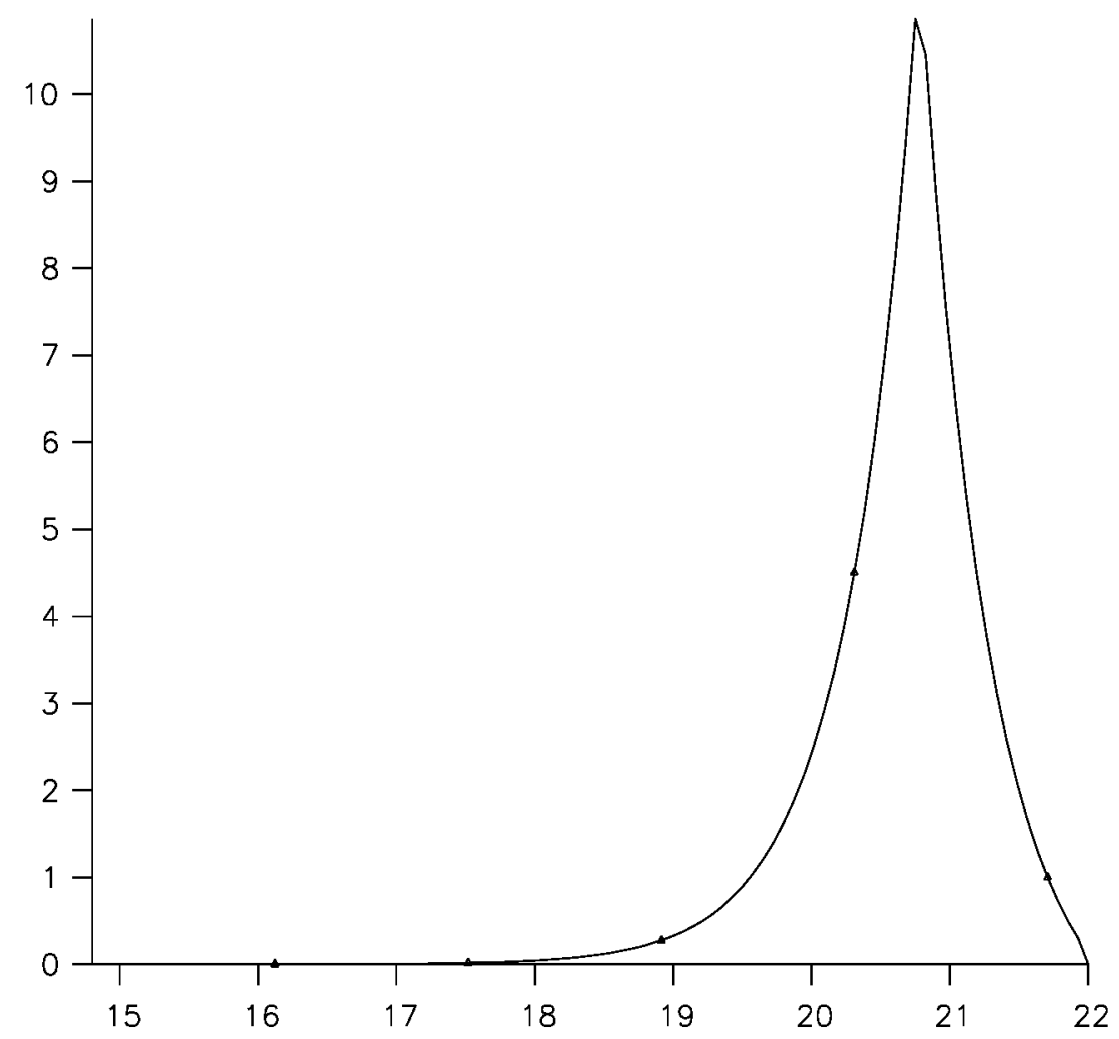

Figure 10: Poloidal velocity $U_{\theta}[\mathrm{km} / \mathrm{sec}]$. The maximum of $u_{\theta}$ is roughly the same as in Fig. 6 analytical theory are less than $\approx 8 \%$. The different profile shape of Fig. 8 effects a rather sharply peaked maximum. 


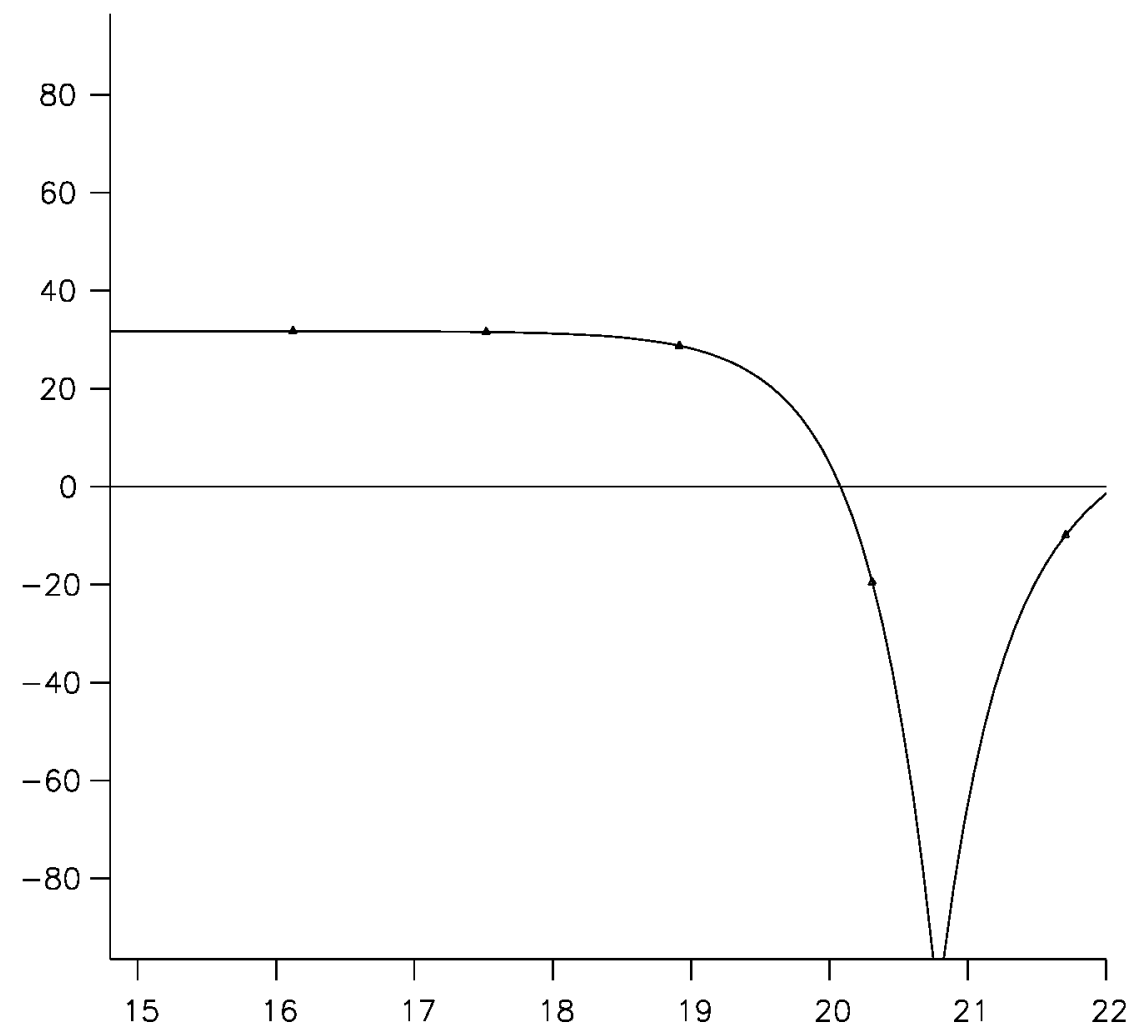

Figure 11: Radial electric field $E_{r}[k V / m]$. The minimum of $E_{r}$ is of the same order as in Fig. 7. Even in the case of a strongly changed temperature profile the electric field is positive at $\mathrm{r}=0$. 


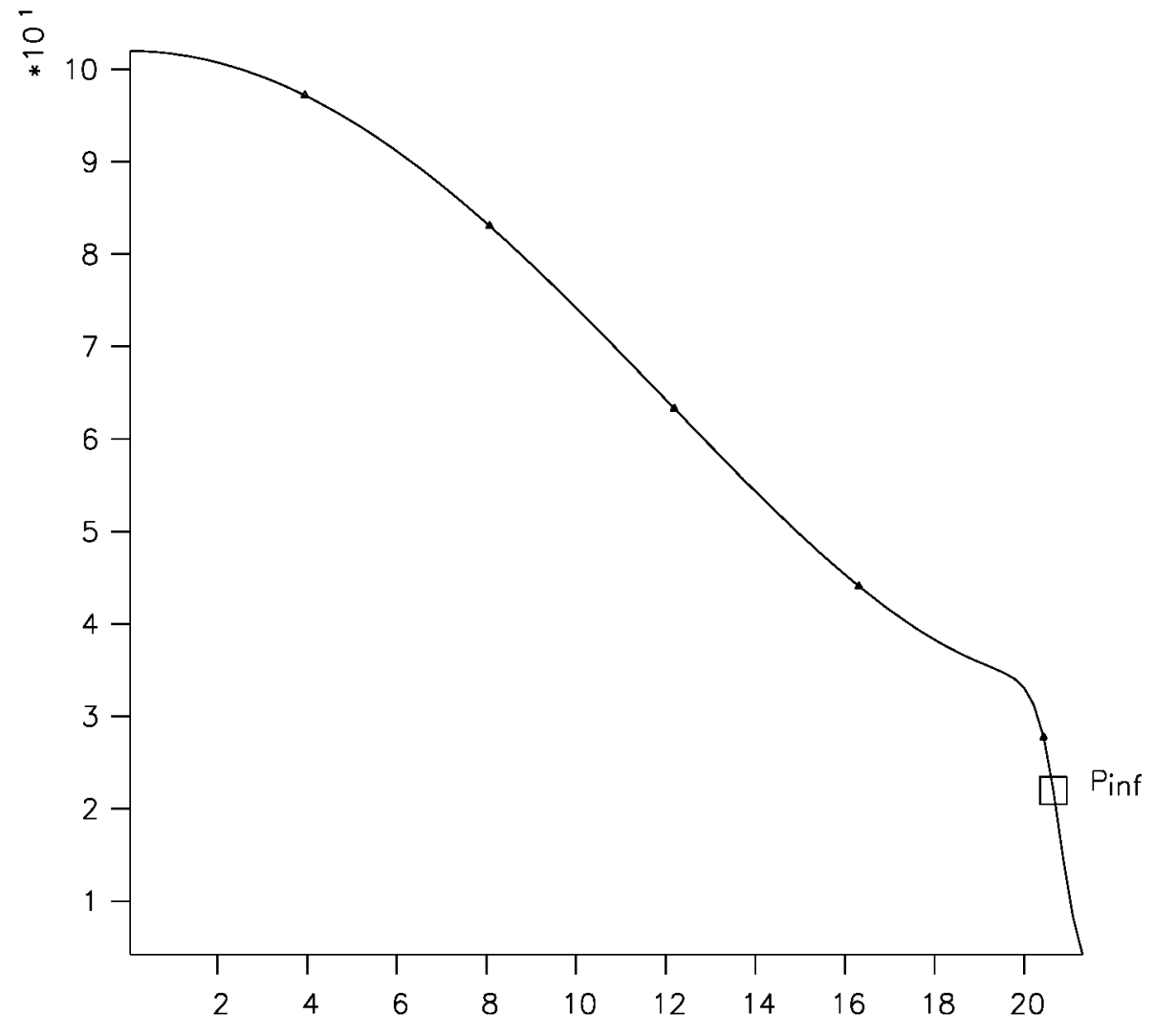

Figure 12: Ion temperature $\mathrm{T}[10 \mathrm{eV}]$. The more realistic temperature profile has a pedestal like in Fig 1 and a parabolic part connecting the edge with the center. 


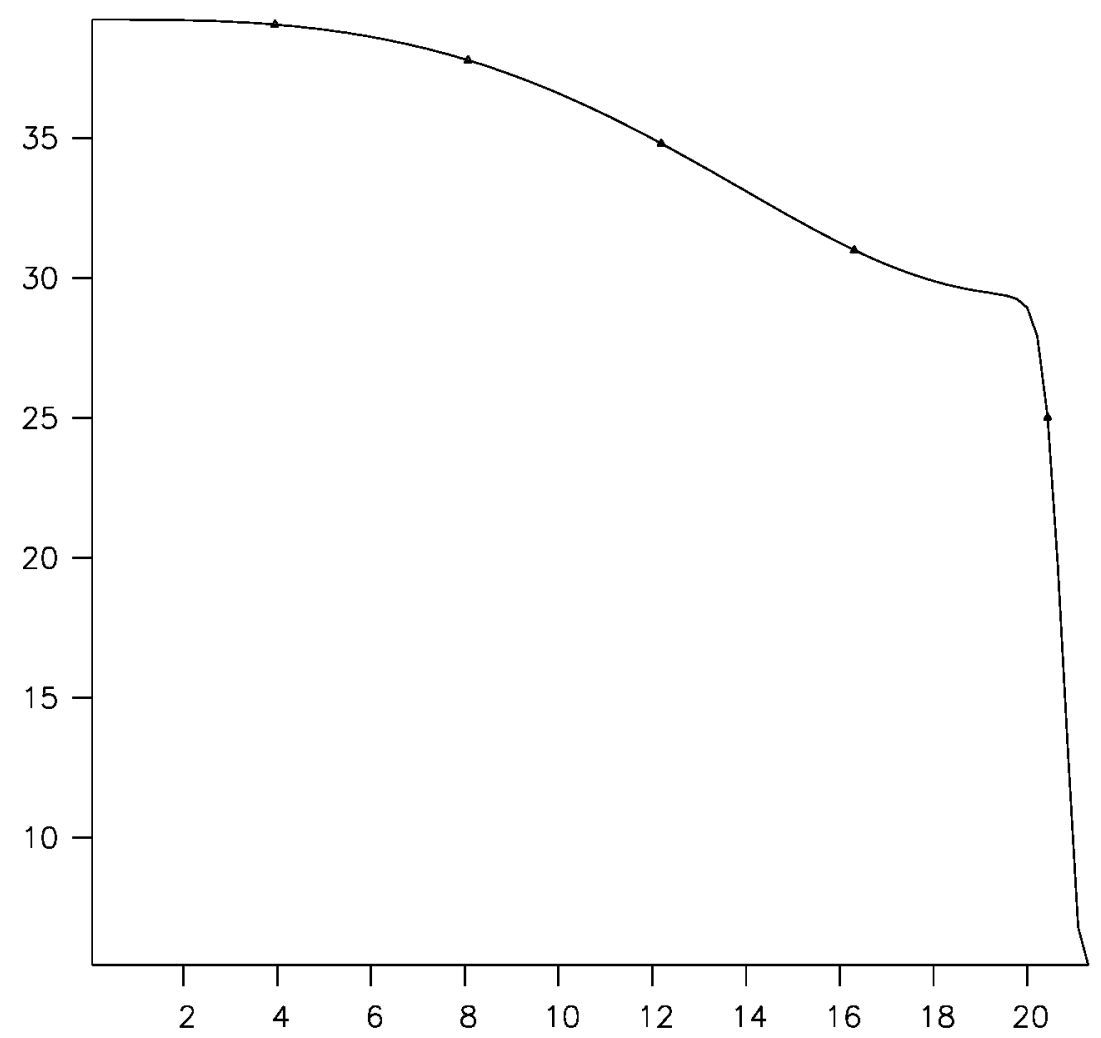

Figure 13: Toroidal velocity $U_{\phi}[\mathrm{km} / \mathrm{sec}]$. The input data are those of ALCATOR C-MOD [30]. The jump $\Delta u_{\phi}$ and the radial dependence of $u_{\phi}$ in the edge are the same as in the corresponding Fig. 5. In going to the center the spin - up increases further because of the temperature gradient. 


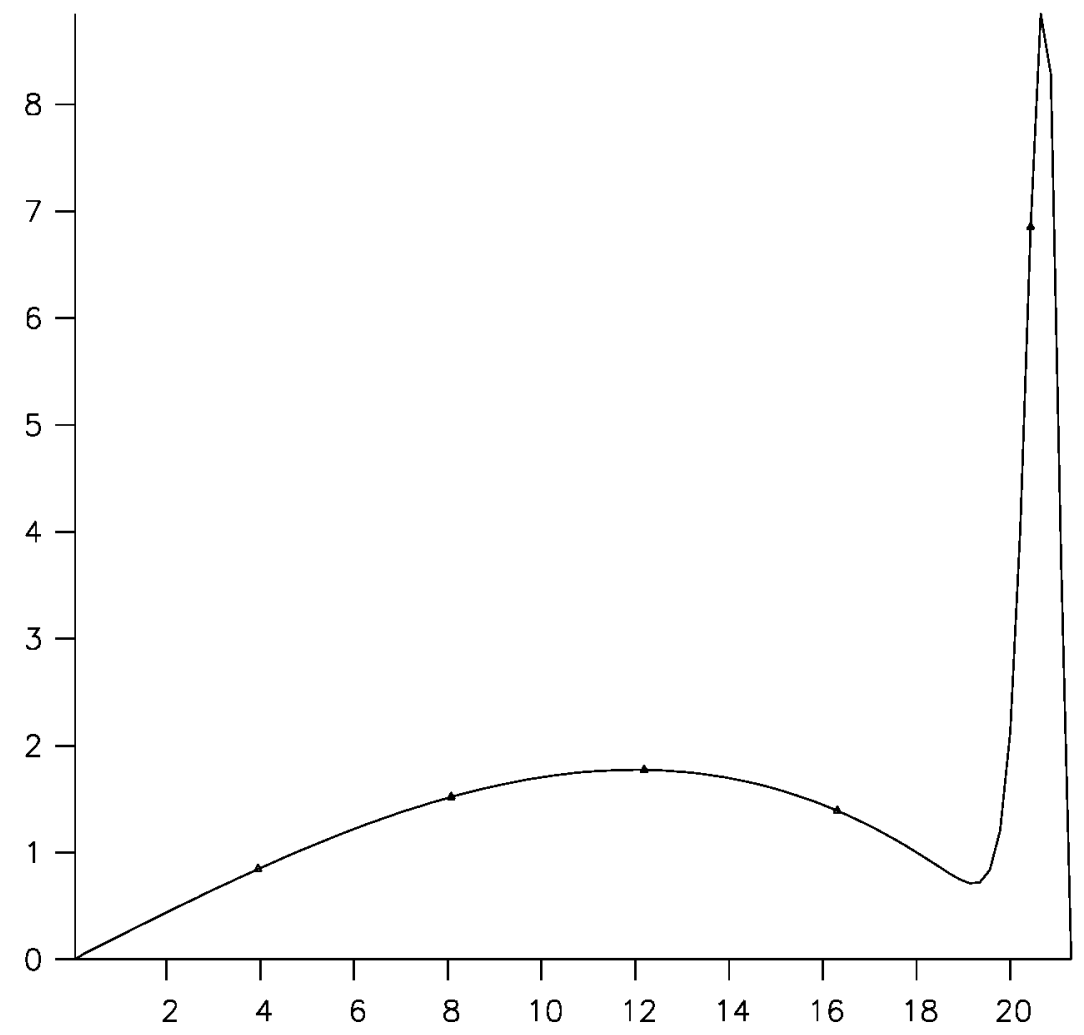

Figure 14: Poloidal velocity $U_{\theta}[\mathrm{km} / \mathrm{sec}]$. The maximum of $u_{\theta}$ due to the pedestal is still pronounced. The poloidal rotation velocity at smaller radii is -according to the temparature gradient - finite but small. 


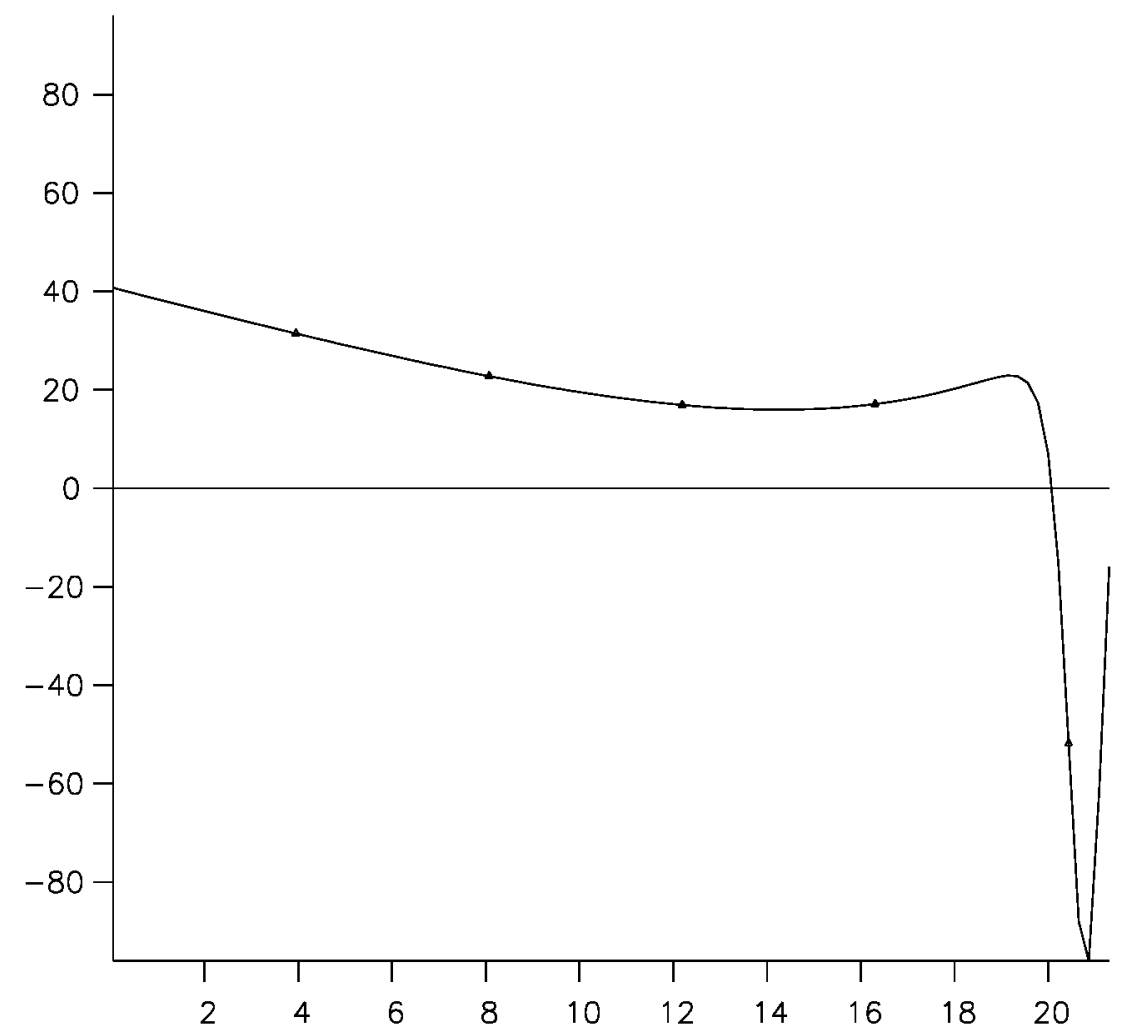

Figure 15: Radial electric field $E_{r}[\mathrm{kV} / \mathrm{m}]$. The electric field deviates at the plasma interior from that of Fig. 7 by at most $20 \%$ 


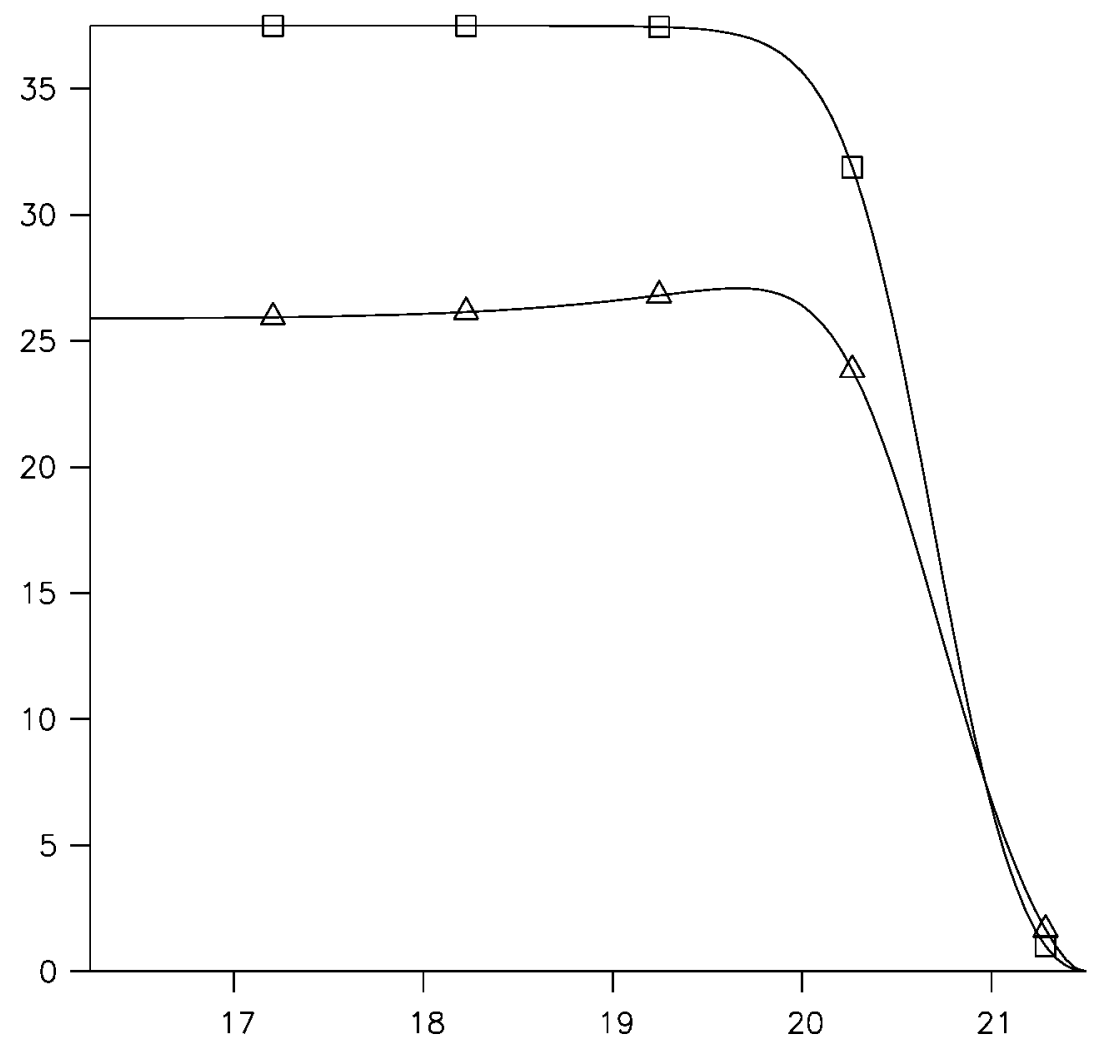

Figure 16: Toroidal velocity $U_{\phi}[\mathrm{km} / \mathrm{sec}$. Even at the very low neutral density of $N_{0}=5 \cdot 10^{14} \frac{1}{m^{3}}$ an effect on the step can be seen which is reduced in size by $25 \%$. 


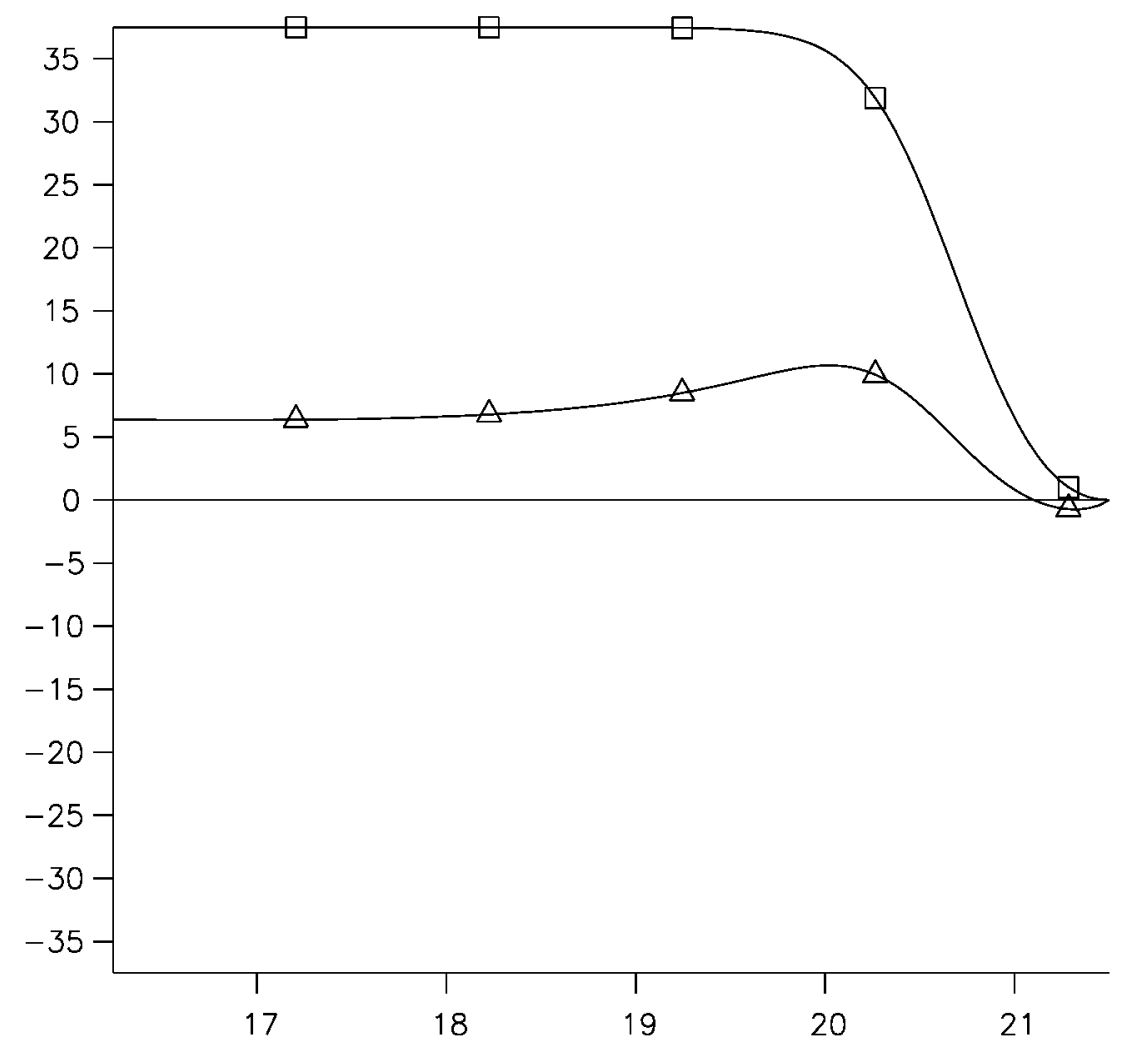

Figure 17: Toroidal velocity $U_{\phi}[\mathrm{km} / \mathrm{sec}]$. If the neutral density $N_{0}$ is increased to $N_{0}=5 \cdot 10^{15} \frac{1}{m^{3}}$, the step in $\mathrm{g}$ is reduced to about $16 \%$, i. e. almost removed. 


\section{References}

[1] F. Wagner, G. Becker, K. Behringer, D. CampbelI, A. Eberhagen, W. Engelhardt, G. Fussmann, O. Gehre, J. Gernhardt, G. V. Gierke, G. Haas, M. Huang, F. Karger, M. Keilhacker, O. Klüber, M. Kornherr, K. Lackner, G. Lisitano, G. G. Lister, H. M. Mayer, D. Meisel, E. R. MülIer, H. Murmann, H. Niedermeyer, W. Poschenrieder, H. Rapp, H. Röhr, F. Schneider, G. Siller, E. Speth, A. Stäbler, K. H. Steuer, G. Venus, O. Vollmer, and Z. Yü, Phys. Rev. Lett. 49,1408 (1982).

[2] ASDEX Team, Nucl. Fusion 29, 1959 (1989).

[3] K. H. Burrell, E. J. Doyle, P. Gohil, R. J. Groebner, J. Kim, R. J. La Haye, L. L. Lao, R. A. Moyer, T. H. Osborne, W. A. Peebles, C. L. Rettig, T. H. Rhodes, and D. M. Thomas, Phys. Plasmas, 1, 1536 (1994).

[4] K. Ida, S. Hidekuma, M. Kojima et al, Phys. Fluids B4, $2552(1992)$

[5] D. P. Schissel, J. C. DeBoo, K. H. Burrell, J. R. Ferron, R. J. Groebner, H. St. John, R. D. Stambaugh, DIII D Research Team, B. J. D. Tubbing, K. Thomsen, J. G. Cordey, M. Keilhacker, D. Stork, P. E. Stott, A. Tanga, and JET Team, Nucl. Fusion 3173 (1991).

[6] C. M. Greenfield, B. Balet, K. H. Burrell, M. S. Chu, J. G. Cordey, J. C. DeBoo, N. Deliyanakis, E. J. Doyle, R. J. Groebner, G. L. Jackson, S. Konoshima, D. P. O'Brien, L. Porte, C. L. Rettig, T. H. Osborne, H. St. John, A. C. 
C. Sips, G.M. Staebler, E. J. Strait, P. M. StubberfieId, T. S. Taylor, S. J. Thompson, K. Thomsen, A. D. Tumbuli, and the JET and DIII - D Teams, Plasma Phys. Control. Fusion 35, B263 (1993).

[7] A. M. Messiaen, J. Ongena, U. Samm, M. Z. Tokar, B. Unterberg, D. Boucher, K. H. Finken, E. Hintz, R. Koch, G. Mank, G. Telesca, P. E. Vandenplas, G. von Oost, G. van Wassenhove, J. Winter, G. H. Wolf, P. Dumortier, F. Durodié, H. G. Esser, H. Euringer, G. Fuchs, D. L. Hulls, F. Hoenen, L. Koenen, H. R. Koslowski, A. Krämer-Flecken, M. Lochter, A. Pospieszczyk, J. Rapp, B. Schweer, H. Soltwisch, R. Uhlemann, R. van Nieuwenhove, M. Vervier, G. Waidmann, and R. R. Weynants, Nucl. Fusion 36, 39 (1996).

[8] F. M. Levinton, M. C. Zarnstoff, S. H. Batha, M. Bell, R. E. Bell, R. V. Budny, C. Bush, Z. Chang, E. Fredrickson, A. Janos, J. Manickam, A. Ramsey, S. A. Sabbagh, G. L. Schmidt, E. J. Synakowski, and G. Taylor, Phys. Rev. Lett. 75,4417 (1995).

[9] E. J. Strait, L. L. Lao, M. E. Mauel, B. W. Rice, T. S. Taylor, K. H. Burrell, M. S. Chu, E. A. Lazarus, T. H. Osborne, S. J. Thompson, and A. D. Turnbull, Phys. Rev. Lett. 75, 4421 (1995).

[10] K. Ushigusa and JT-60 Team, in Fusion Energy, 1996, Montreal (International Atomic Energy Agency, Vienna, 1997), Vol.1, p.37. 
[11] Equipe Tore Supra, in Fusion Energy, 1996, Montreal (International Atomic Energy Agency, Vienna, 1997), Vol.1, p.141.

[12] ITER Joint Central Team, Plasma Phys. Control. Fusion 35, B23 (1993).

[13] S.-I. ltoh and K. ltoh, Nucl. Fusion 29,1031(1989).

[14] K. C. Shaing, E. C. Crume, Jr., and W. A. Houlberg, (Phys. Fluids B2 1492 (1990).

[15] V. Rozhansky and M. Tendler, Phys. Fluids B4 1877 (1992).

[16] F. L. Hinton, J. Kim, Y.-B. Kim, A. Brizard, and K. H. Burrell, Phys. Rev. Lett. 72, 1216 (1994).

[17] P. H. Diamond, Y. - M. Liang, B. A. Carreras, and P. W. Terry, Phys. Rev. Lett 722565 (1994).

[18] S. J. Braginski, Transport processes in a plasma, Review of Plasma Physics, New York, Consultants Bureau, vol. 1, p. 205

[19] T. E. Stringer, Phys. Rev. Letters, 22 (1969) 770

[20] M. N. Rosenbluth, J. B. Taylor, Phys. Rev. Letters, 23 (1969) 367

[21] A. B. Hassam, T. M. Antonsen, Jr., Phys. Plasmas, 1, No. 2 (1994) 337

[22] A. Rogister, Phys. Plasmas, 1, 619, (1994) 
[23] A. Rogister, Fusion Energy, Montreal (International Atomic Energy Agemcy, Vienna (1977)) Vol 2 p.573 (1996)

[24] A. Rogister, Phys. Rev. Lett. 81, 3663 (1998)

[25] H. A. Claassen, H. Gerhauser, A. Rogister, C. Yarim, Physics of Plasmas, 7 (2000) 3699

[26] A. B. Mikhailowski, A. B. Tsypin, V. S., Beiträge zur Plasmaphysik, 24 (1984) 335.

[27] H. A. Claaßen, H. Gerhauser, Fusion Theory, Proceedings of the 7th European Fusion Theory Conference p. 199

[28] W. H. Press, S. A. Teukolsky, W. T. Vetterling, B. R. Flannery Numerical Recipes in FORTRAN, Cambridge University Press (1992)

[29] A. L. Rogister, J. E. Rice, A. Nicolai, P. T. Bonoli, M. J. Greenwald \& ALCATOR C-MOD Group, Proceedings of the 28 EPS, paper P3.034, 2001

[30] J. E. Rice et al., Phys. Plasmas 7 (2000) 1825

[31] K. Audenaerde, G.A. Emmert, and M. Gordinier, University of Wisconsin, Report UWFDM-259, 1978

[32] U. Daybelge, Proceedings of the 18th International Conference on Fusion Energy 2000, Sorrento, Italy, paper THP $1 / 21$

[33] A. V. Chankin, G. Corrigan, S. K. Erents, G. F. Matthews, J. Spence, P. C. Stangeby, J. Nucl. Mat. 290-293 (2001) 518 


\section{A Temperature and density profiles with $L_{\psi}$ as decay length}

Inside the last closed flux surface with radius $r_{s}$ an exponential decay hat been assumed for the temperature and density profile.

$$
\hat{T}=\hat{T}_{0}+\left(1-\hat{T}_{0}\right) \exp \left(-\frac{r-r_{i n f}}{L_{\psi}\left(1-\hat{T}_{0}\right)}\right)
$$

or

$$
\hat{T}=\hat{T}_{0}+\left(1-\hat{T}_{0}\right) \exp \left(-\frac{x}{1-\hat{T}_{0}}\right)
$$

with $\hat{T}(r=0)=\hat{T}_{0}$ and $\hat{T}\left(r=r_{\text {inf }}\right)=1 . L_{\psi}$ is related to the temperature scale - length $L_{T s}$ (see below). Analogously we have for the density profile

$$
\hat{n}=\hat{n}_{0}+\left(1-\hat{n}_{0}\right) \exp \left(-\frac{r-r_{i n f}}{L_{\psi_{n}}\left(1-\hat{n}_{0}\right)}\right)
$$

or with $r-r_{i n f}=L_{\psi} x$

$$
\hat{n}=\hat{n}_{0}+\left(1-\hat{n}_{0}\right) \exp \left(-\frac{L_{\psi}}{L_{\psi_{n}}\left(1-\hat{n}_{0}\right)} x\right)
$$

with $\hat{n}(r=0)=\hat{n}_{0}$.

$\hat{T}_{0}, \hat{n}_{0}$ are the central values and $r_{\text {inf }}$ is the radius of the inflection point. $L_{\psi_{n}}$ is related to the density scale length in an analogous way as is $L_{\psi}$ to the temperature scale length.

The first and second derivative have the simple forms

$$
\begin{gathered}
\frac{d \hat{T}}{d x}=-\exp \left(-\frac{x}{1-\hat{n}_{0}}\right) \\
\frac{d \hat{n}}{d x}=-\frac{L_{\psi}}{L_{\psi_{n}}} \exp \left(-\frac{L_{\psi}}{L_{\psi_{n}}} \frac{x}{1-\hat{n}_{0}}\right)
\end{gathered}
$$


and

$$
\begin{gathered}
\frac{d^{2} \hat{T}}{d x^{2}}=\frac{1}{1-\hat{T}_{0}} \exp \left(\frac{-x}{1-\hat{T}_{0}}\right) \\
\frac{d^{2} \hat{n}}{d x^{2}}=\left[\frac{L_{\psi}}{L_{\psi_{n}}}\right]^{2} \frac{1}{1-\hat{n}_{0}} \exp \left(-\frac{L_{\psi}}{L_{\psi_{n}}} \frac{x}{1-\hat{n}_{0}}\right)
\end{gathered}
$$

At the inflection point we have

$$
\begin{gathered}
\left(\frac{d \hat{T}}{d x}\right)_{x=0}=-1 \\
\left(\frac{d \hat{n}}{d x}\right)_{x=0}=-\frac{L_{\psi}}{L_{\psi_{n}}}
\end{gathered}
$$

The temperature and density scale lengths are

$$
\begin{gathered}
L_{T}=\frac{T}{\frac{\partial T}{\partial r}}=\frac{L_{\psi} \hat{T}}{\frac{d \hat{T}}{d x}}=L_{\psi}\left[\frac{\hat{T}_{0}}{-\exp \left(-\frac{x}{1-\hat{T}_{0}}\right)}-\left(1-T_{0}\right)\right] \\
\begin{aligned}
L_{n}=\frac{n}{\frac{\partial n}{\partial r}} & =\frac{L_{\psi} \hat{n}}{\frac{d \hat{n}}{d x}}=\frac{L_{\psi}\left[\hat{n}_{0}+\left(1-\hat{n}_{0}\right) \exp \left(-\frac{r-r_{i n f}}{L_{\psi_{n}}\left(1-\hat{n}_{0}\right)}\right)\right.}{-\frac{L_{\psi}}{L_{\psi_{n}}} \exp \left(-\frac{L_{\psi}}{L_{\psi_{n}}} \frac{x}{1-\hat{n}_{0}}\right)} \\
& =L_{\psi_{n}}\left[\frac{\hat{n}_{0}}{-\exp \left(-\frac{L_{\psi}}{L_{\psi_{n}}} \frac{x}{1-\hat{n}_{0}}\right)}-\left(1-\hat{n}_{0}\right)\right]
\end{aligned}
\end{gathered}
$$

respectively.

The temperature and density scale lengths at the inflection point are then

$$
\begin{aligned}
L_{T_{i n f}} & =\left(\frac{T}{\frac{\partial T}{\partial r}}\right)_{i n f}=-L_{\psi} \\
L_{n_{i n f}} & =\left(\frac{n}{\frac{\partial n}{\partial r}}\right)_{i n f}=-L_{\psi_{n}}
\end{aligned}
$$


They are then independent from the maximum values $\hat{T}_{0}$ and $\hat{n}_{0}$.

Outside the last closed flux surface also an exponential decay hat been assumed for the temperature and density profile. For the temperature we have

$$
\hat{T}=\exp \left[\frac{r-r_{i n f}}{L_{\psi}}\right]
$$

or

$$
\hat{T}=\exp [-x]
$$

with $\hat{T}(x=0)=1$ and $\hat{T}(r=\infty)=0$. The first and second derivative have the simple form

$$
\begin{aligned}
& \frac{d \hat{T}}{d x}=-\exp [-x] \\
& \frac{d^{2} \hat{T}}{d x^{2}}=\exp [-x]
\end{aligned}
$$

The temperature scale length is

$$
\frac{T}{\frac{\partial T}{\partial r}}=\frac{L_{\psi} \hat{T}}{\frac{d \hat{T}}{d x}}=-L_{\psi}
$$

It is a constant and equal to $\left(\frac{T}{\frac{\partial T}{\partial r}}\right)_{r=r_{i n f}}$ so that the radial temperature dependance is continuous and 'smooth' at $\mathrm{r}=r_{\text {inf }}$. (the curvature, however, is discontinuous.)

For the density analogous formulae are valid. In the calculations we assume $L_{\psi_{n}}=L_{\psi}$. Thus we have utilized the same model behaviour for both $\mathrm{T}$ and $\mathrm{n}$ with the same scale length $L_{\psi}$. 


\section{B Ion - ion collisionality and collision fre- quency}

In the following we collect some standard formulas for the collisionality and the collision frequency. For the collisionality $\hat{\nu}_{i}$ we have $\left(N_{i}=\frac{n_{i}}{10^{13}}, N_{\text {inf }}=\frac{n_{i n f}}{10^{13}}\right)$

$$
\hat{\nu}_{i}=\frac{q R}{c_{i} \tau_{i}}=7.1410^{2} \frac{q R N_{i} Z_{e f f_{i}}}{T_{i}^{2}}
$$

With the thermal speed

$$
c_{i}=\sqrt{\frac{k T_{i}}{A}}=0.9910^{4} \sqrt{\frac{T_{i}[\mathrm{eV}]}{m_{i}}} \frac{m}{\mathrm{sec}}
$$

we get the collision frequency

$$
\nu_{i}=\frac{1}{\tau_{i}}=7.1410^{2} \frac{N_{i} Z_{\text {eff }}}{T_{i}^{2}} 0.9910^{4} \sqrt{\frac{T_{i}[\mathrm{eV}]}{A}}
$$

or

$$
\nu_{i}=\frac{1}{\tau_{i}}=7.1410 \frac{N_{i} Z_{e f f_{i}}}{T_{i}^{\frac{3}{2}} \sqrt{A}}
$$

The collision time is then

$$
\tau_{i}=\frac{1}{\nu_{i}}=\frac{1.414}{7.14} 10^{-6} \frac{T_{i}^{\frac{3}{2}} \sqrt{\frac{A}{2}}}{N_{i} Z_{e f f_{i}}}
$$

or

$$
\tau_{i}=\frac{1}{\nu_{i}}=1.9810^{-7} \frac{T_{i}^{\frac{3}{2}} \sqrt{\frac{A}{2}}}{N_{i} Z_{\text {eff }}}
$$

This formula compares well with that of Braginski if a Coulomb $\operatorname{logarithm} \lambda_{\text {coul }}=15$ is assumed. More exactly we have

$$
\tau_{i}=\frac{1}{\nu_{i}}=\frac{1.9810^{6}}{\left(\frac{\lambda_{\text {coull }}}{15}\right)} T_{i}^{\frac{3}{2}} \sqrt{\frac{A}{2}} n_{i} Z_{\text {eff }}
$$




\section{Parameter $\Lambda_{1}$}

The connection of $\Lambda_{1}$ with the velocity $\mathrm{S}$ is given by

$$
\Lambda_{1}=\frac{2 \tilde{v}_{T}}{S} \frac{B_{\phi}}{B}
$$

$\mathrm{S}$ is defined by

$$
S=\frac{8 r c_{i}}{\hat{\nu}_{i} q R}
$$

Therefore

$$
\Lambda_{1}=\frac{2 \tilde{v}_{T}}{\frac{8 r c_{i}}{\hat{\nu}_{i} q R}} \frac{B_{\phi}}{B}
$$

where $\tilde{v}_{T}=\frac{1}{e B_{\phi}} \frac{T}{L_{T}}$ with $L_{T}=\frac{T}{\frac{\partial l n}{\partial r}}$. Therefore we get

$$
\Lambda_{1}=\frac{2 \frac{1}{e B_{\phi}} \frac{T}{L_{T}}}{\frac{8 c_{i}}{\hat{\nu}_{i} q R}} \frac{B_{\phi}}{B}=\frac{\nu_{i}}{\Omega_{i}} \frac{q^{2} R^{2}}{L_{T} 4 r}
$$

At the inflection point we have $\left(L_{T}=L_{T, \text { inf }}\right)$

$$
\Lambda_{1, i n f}=\frac{2 \frac{1}{e B_{\phi}} \frac{T_{i n f}}{L_{T, i n f}}}{\frac{8 r_{i n f} c_{i n f}}{q q_{i n f} \tau_{i, i n f}} q R} \frac{B_{\phi}}{B}
$$

or

$$
\Lambda_{1, i n f}=2 \frac{1}{e B_{\phi}} \frac{T_{i n f}^{2} R^{2}}{L_{T, i n f} 8 r_{i n f} c_{i n f}^{2} \tau_{i i, i n f}}
$$

with $\Omega_{i}=\frac{e B}{m_{i}}$ we get

$$
\Lambda_{1, i n f}=\frac{\nu_{i i, i n f}}{\Omega_{i}} \frac{q^{2} R^{2}}{L_{T, i n f} 4 r_{i n f}}
$$


The quantity $\Lambda_{1, \text { inf }}^{\prime}$ used in appendix $\mathrm{G}$ is defined by

$$
\Lambda_{1, i n f}^{\prime}=\frac{\nu_{i i, i n f}}{\Omega_{i}} \frac{q^{2} R^{2}}{L_{\psi} 4 r_{i n f}}=\frac{L_{T, i n f}}{L_{\psi}} \Lambda_{1, i n f}=-\Lambda_{1, i n f}
$$

for $L_{T, \text { inf }}=-L_{\psi}$ as in the case of the preceding temperature profiles.

The radial dependence due to the plasma parameters is given by

or

$$
\Lambda_{1}=\Lambda_{1, i n f} \hat{n} \hat{T}^{-\frac{3}{2}} Z_{e f f} \frac{r_{i n f}}{r} L_{T, i n f} \frac{d l n(T)}{d r}
$$

$$
\Lambda_{1}=\Lambda_{1}^{\prime} \frac{L_{\psi}}{L_{T}}
$$

$\Lambda_{1}^{\prime}$ is given by

$$
\Lambda_{1}^{\prime}=\Lambda_{1} \frac{L_{T}}{L_{\psi}}=\frac{\nu_{i i}}{\Omega_{i}} \frac{q^{2} R^{2}}{L_{\psi} 4 r}
$$

we assume $\hat{n}=\hat{T}^{\frac{1}{\eta}}$ and $\hat{Z}_{\text {eff }}=\hat{T}^{Z}$ where $\eta$ can be written as

$$
\eta=\frac{\frac{d \ln (\hat{T})}{d x}}{\frac{d \ln (\hat{n})}{d x}}
$$

We get (appendix B)

$$
\Lambda_{1}=\Lambda_{1, \text { inf }} \frac{1}{\hat{T}^{\frac{3}{2}-\frac{1}{\eta}-Z}} \frac{r_{\text {inf }}}{r} L_{T, \text { inf }} \frac{d \ln (T)}{d r}
$$

or

$$
\Lambda_{1}=\Lambda_{1, i n f} \frac{1}{\hat{T}^{\frac{5}{2}-\frac{1}{\eta}-Z}} \frac{r_{\text {inf }}}{r} \frac{d \hat{T}}{d x}
$$

If we assume that the exponent of $\hat{T}, \frac{5}{2}-\frac{1}{\eta}-Z$, is equal to zero, we get

$$
\Lambda_{1}=\Lambda_{1, i n f} \frac{r_{\text {inf }}}{r} L_{T, i n f} \frac{d l n(T)}{d r}
$$


or

$$
\Lambda_{1}=\Lambda_{1, i n f} \frac{r_{i n f}}{r} \frac{L_{T, i n f}}{L_{T}}
$$

It is sometimes convenient to introduce the quantity $\Lambda_{r}$. We use the expression

$$
\Lambda_{1}=\frac{2 \tilde{v}_{T}}{S} \frac{B_{\phi}}{B}
$$

and replace $\mathrm{S}$

$$
\Lambda_{1}=2 \tilde{v}_{T} \frac{\hat{\nu}_{i} q R}{8 r c_{i}} \frac{B_{\phi}}{B}
$$

Then we replace $\tilde{v}_{T}$ and get

$$
\Lambda_{1}=2 \frac{T}{e B_{\phi} L_{T}} \frac{\hat{\nu}_{i} q R}{8 r c_{i}} \frac{B_{\phi}}{B}
$$

We use $q=\frac{r B_{\phi}}{R B_{\theta}}, T=m_{i} c_{i}^{2}, e B=m_{i} \Omega_{i}$ and obtain

$$
\Lambda_{1}=2 \frac{m_{i} c_{i}^{2}}{m_{i} \Omega_{i} L_{T}} \frac{\hat{\nu}_{i} r R}{R B_{\theta} 8 r c_{i}} \frac{1}{B}
$$

With $a_{i}=\frac{c_{i}}{\Omega_{i}}$ we get

$$
\Lambda_{1}=\frac{a_{i}}{L_{T}} \frac{\hat{\nu}_{i}}{B_{\theta} 4} B_{\phi}
$$

We define $\Lambda_{r}=4 \Lambda_{1}$ and obtain

$$
\Lambda_{r}=\frac{a_{i}}{L_{T}} \frac{\hat{\nu}_{i}}{B_{\theta}} B_{\phi}
$$

With $a_{p_{i}}$, the Larmorradius in the poloidal field we get the simple expression

$$
\Lambda_{r}=\frac{a_{p_{i}}}{L_{T}} \hat{\nu}_{i}
$$




\section{Reciprocal time $\eta^{\prime}$}

The quantity $\eta^{\prime}$, is defined as

$$
\eta^{\prime}=\frac{1}{m_{i} n_{i n f} L_{\psi}^{2}} \eta_{2}=\frac{\frac{6}{5} N_{i n f} k T \tau_{i i}}{\Omega_{i}^{2} \tau_{i i}^{2} m_{i} n_{i} L_{\psi}^{2}}
$$

With the expression for the thermal speed $c_{i}$ we get

$$
\eta^{\prime}=\frac{\frac{6}{5} c_{i}^{2}}{\Omega_{i}^{2} \tau_{i i} L_{\psi}^{2}}
$$

Replacing $\tau_{i i}$ by means of the collisionality yields

$$
\eta^{\prime}=\frac{\frac{6}{5} c_{i}^{2}}{\Omega_{i}^{2} L_{\psi}^{2}} \frac{\hat{\nu}_{i} c_{i}}{q R}
$$

With the expressions for the collisionality and the thermal speed we get

$$
\eta^{\prime}=\frac{\frac{6}{5} c_{i}^{2}}{\Omega_{i}^{2} L_{\psi}^{2}} \frac{7.1410^{2} \frac{q R N_{i} Z_{e f f_{i}}}{T_{i}^{2}} c_{i}}{q R}
$$

Introducing the Larmorradius $a_{i}=\frac{c_{i}}{\Omega_{i}}$ gives

$$
\eta^{\prime}=\frac{\frac{6}{5} a_{i}^{2}}{L_{\psi}^{2}} \frac{7.1410^{2} \frac{q R N_{i} Z_{e f f_{i}}}{T_{i}^{2}} 0.9910^{4} \sqrt{\frac{T_{i}[e V]}{A}}}{q R}
$$

or

$$
\eta^{\prime}=\frac{\frac{6}{5} a_{i}^{2}}{L_{\psi}^{2}} 714 \frac{N_{i} Z_{\text {eff }}}{T_{i}^{\frac{3}{2}}} 0.9910^{4} \sqrt{\frac{1}{A}}
$$

and finally

$$
\eta^{\prime}=8.5710^{-7} \frac{a_{i}^{2}}{L_{\psi}^{2}} \frac{n_{i} Z_{\text {eff }}}{T_{i}^{\frac{3}{2}}} \sqrt{\frac{1}{A}}
$$


Replacing $\tau_{i i}$ by means of $\Lambda_{1}$ we get

$$
\eta^{\prime}=\frac{\frac{6}{5} c_{i}^{2}}{\Omega_{i}^{2} L_{\psi}^{2}}\left|\Lambda_{1}\right| \frac{L_{\psi} 4 r}{q^{2} R^{2}} \Omega_{i}
$$

or, simplifying

$$
\eta^{\prime}=\frac{\frac{6}{5} a_{i}^{2}}{L_{\psi}^{2}}\left|\Lambda_{1}\right| \frac{L_{\psi} 4 r}{q^{2} R^{2}} \Omega_{i}
$$

$a_{i}$ is the Larmorradius. Outside (inside) the inflection point we have

$$
\eta^{\prime}=\eta_{\text {inf }}^{\prime} \hat{T}^{-\frac{1}{2}} \hat{n}
$$

with

$$
\eta_{\text {inf }}^{\prime}=\frac{\frac{6}{5} a_{i}^{2}}{L_{\psi}^{2}}\left|\Lambda_{1, i n f}\right| \frac{L_{\psi} 4 r_{i n f}}{q^{2} R^{2}} \Omega_{i}
$$




\section{E Velocity S}

We use two expressions giving the exact result, the first resorts to $\chi_{\|}$and the second to $\Lambda_{1}$. The first is

$$
\frac{S}{\tilde{v}_{T}}=\frac{2 r \chi_{\|}}{4 n q^{2} R^{2} \tilde{v}_{T}}
$$

We use

$$
\chi_{\|}=4 c^{2} n \tau_{i i}=\frac{4 T_{i} n \tau_{i i}}{m_{i}}
$$

and get

$$
\frac{S}{\tilde{v}_{T}}=\frac{2 r \chi_{\|}}{n q^{2} R^{2} \tilde{v}_{T}}=\frac{2 r 4 T_{i}}{m_{i} \nu_{i i} q^{2} R^{2} \tilde{v}_{T}}
$$

or with $\tilde{v}_{T}=\frac{T}{e B_{\phi}} \frac{\partial \ln T}{\partial r}$

$$
\frac{S}{\tilde{v}_{T}}=\frac{2 r 4 T_{i} n}{m_{i} \nu_{i i} n q^{2} R^{2} \tilde{v}_{T}}=\frac{\Omega_{i} L_{T} 8 r}{\nu_{i i} q^{2} R^{2}}=\frac{2}{\Lambda_{1}}
$$

At the inflection point we have

$$
\frac{S_{i n f}}{\tilde{v}_{T, i n f}}=\frac{2 r_{i n f} 4 T_{i n f} n}{m_{i} \nu_{i i, i n f} n q^{2} R^{2} \tilde{v}_{T, s}}=\frac{\Omega_{i} L_{T, i n f} 8 r_{i n f}}{\nu_{i i, i n f} q^{2} R^{2}}=\frac{2}{\Lambda_{1, i n f}}
$$

or with $v_{T}=\frac{1}{e B_{\phi}} \frac{T_{i n f}}{L_{\psi}}$

$$
\frac{S_{i n f}}{v_{T}}=\frac{2 r_{i n f} 4 T_{i n f} n}{m_{i} \nu_{i i, i n f} n q^{2} R^{2} v_{T}}=\frac{\Omega_{i} L_{\psi} 8 r_{i n f}}{\nu_{i i, i n f} q^{2} R^{2}}=\frac{2}{\Lambda_{1, i n f}}
$$

Alternatively the formula

$$
\frac{S_{i n f}}{v_{T}}=\frac{2}{\Lambda_{1, i n f}^{\prime}}
$$


can be employed which is analytically identical with the aforementioned formula. The scaling with the plasmaparameters is given by

$$
\begin{gathered}
\frac{S}{v_{T}}=\frac{S}{\tilde{v}_{T}} \frac{\partial \hat{T}}{\partial x}=\frac{S}{\tilde{v}_{T}} L_{\psi} \frac{\partial \hat{T}}{\partial r}=\frac{S}{\tilde{v}_{T}} L_{\psi} \frac{\partial \hat{T}}{\frac{\partial r}{\hat{T}}} \hat{T}= \\
\frac{S}{\tilde{v}_{T}} \frac{L_{\psi}}{L_{T}} \hat{T}=\frac{S_{i n f}}{v_{T}} \frac{\hat{T}^{\frac{3}{2}}}{\hat{n}} \frac{L_{\psi}}{L_{T}} \hat{T} \\
\frac{S}{v_{T}}=\frac{S_{\text {inf }}}{v_{T}} \frac{\hat{T}^{\frac{3}{2}}}{\hat{n}} \\
\frac{S}{4 v_{T}}=\frac{1}{2 \Lambda_{1, i n f}} \frac{\hat{T}^{\frac{3}{2}}}{\hat{n}}
\end{gathered}
$$

We also have

$$
\frac{S}{\tilde{v}_{T}}=\frac{2}{\Lambda_{1}} \frac{B_{\phi}}{B}=\frac{8}{\Lambda_{r}} \frac{B_{\phi}}{B}
$$

and

$$
\frac{S}{v_{T}}=\frac{2}{\Lambda_{1}} \frac{B_{\phi}}{B} \frac{\tilde{v}_{T}}{v_{T}}=\frac{2}{\Lambda_{1}} \frac{B_{\phi}}{B} \frac{\mathrm{E}_{\psi}}{L_{T}}=\frac{2}{\Lambda_{1}^{\prime}} \frac{B_{\phi}}{B}
$$

\section{F Velocity Q}

$\mathrm{Q}$ is defined in [22] and is given by

$$
\begin{aligned}
& Q=\left(4 u_{\theta}-5 v_{n}-2.5 \tilde{v}_{T}\right) \frac{B_{\phi}}{B} \\
& Q=\left(4 u_{\theta}-5 v_{n}\left(1+\frac{1}{2 \eta}\right)\right) \frac{B_{\phi}}{B}
\end{aligned}
$$

with the neoclassical value $u_{\theta}=\kappa \frac{\partial T}{\partial x} v_{T}$

$$
Q=\left(4 \kappa \frac{\partial T}{\partial x} v_{T}-5 v_{n}\left(1+\frac{1}{2 \eta}\right)\right) \frac{B_{\phi}}{B}
$$


We get by normalizing

$$
\frac{Q}{4 v_{T}}=h-\hat{T} \frac{\partial \ln \left(n^{2} T\right)}{\partial x} \frac{2.5}{4}
$$

and

$$
\frac{Q}{\tilde{v}_{T}}=\left(4 h^{\prime}-\frac{5}{\eta}-2.5\right) \frac{B_{\phi}}{B}
$$

$h^{\prime}$ is defined by

$$
h^{\prime}=\frac{u_{\theta}}{\tilde{v}_{T}}
$$

Using the neoclassical expression

$$
u_{\theta}=\frac{\kappa}{e B} \frac{\partial T}{\partial r}
$$

and

$$
h^{\prime}=\frac{\kappa}{\frac{\kappa}{e B} \frac{\partial T}{\partial r}} \tilde{v}_{T}=\kappa
$$

we get

$$
\frac{Q}{\tilde{v}_{T}}=\left(4 \kappa-\frac{5}{\eta}-2.5\right) \frac{B_{\phi}}{B}
$$

It follows the derivative $\frac{\partial Q}{\partial h}=4 v_{T}$. We get the ratio

$$
\begin{aligned}
& \frac{Q}{S}=\frac{h-\hat{T} \frac{\partial \ln \left(n^{2} T\right)}{\partial x} \frac{2.5}{4}}{\frac{1}{2 \Lambda_{1}^{\prime}} \frac{B_{\phi}}{B}} \\
& \frac{Q}{S}=\frac{h-\hat{T} \frac{\partial \ln \left(n^{2} T\right)}{\partial x} \frac{2.5}{4}}{\frac{S_{i n f}}{4 v_{T}} \frac{\hat{T}^{\frac{3}{2}}}{\hat{n}} \frac{B_{\phi}}{B}}
\end{aligned}
$$

We also get by using $\mathrm{Q}$ and $\mathrm{S}$ normalized to $\tilde{v}_{T}$

$$
\frac{Q}{S}=\frac{\left(4 h^{\prime}-\frac{5}{\eta}-2.5\right) \frac{B_{\phi}}{B}}{\frac{8}{\Lambda_{r}} \frac{B_{\phi}}{B}}
$$


or with $\frac{2.5}{8}=0.3125$

$$
\begin{aligned}
& \frac{Q}{S}=\frac{\left(0.5 h^{\prime}-0.3125\left(1+\frac{2}{\eta}\right)\right) \frac{B_{\phi}}{B}}{\frac{1}{\Lambda_{r}} \frac{B_{\phi}}{B}} \\
& \frac{Q}{S}=\left(0.5 h^{\prime}-0.3125\left(1+\frac{2}{\eta}\right)\right) \Lambda_{r} \\
& \frac{Q}{S}=0.5\left(h^{\prime}-0.625\left(1+\frac{2}{\eta}\right)\right) \Lambda_{r}
\end{aligned}
$$




\section{G Evaluation of $T_{36}$}

Two exact expressions are given, the first involves the collisionfrequency and the second $\Lambda_{1}$.

\section{G.1 1st expression}

In the first case we have

$$
T_{36}=0.36 \frac{\eta_{1 s}}{\eta_{0 s}\left(1+\frac{Q^{2}}{S^{2}}\right)} q^{2} \frac{1}{\hat{T} v_{T}} \frac{R^{2}}{L_{\psi}^{2}}\left(\frac{\partial \ln T}{\partial x}\right)
$$

or

$$
T_{36}=0.36 \frac{6}{50.96} \frac{\nu_{i i, s}^{2}}{\Omega^{2}} \frac{1}{\left(1+\frac{Q^{2}}{S^{2}}\right)} q^{2} \frac{1}{\hat{T} v_{T}} \frac{R^{2}}{L_{\psi}^{2}}\left(\frac{\partial \ln T}{\partial x}\right)
$$

\section{G.2 2nd expression}

We introduce

$$
\Lambda_{1}^{\prime}=\frac{\nu_{i}}{\Omega_{i}} \frac{q^{2} R^{2}}{L_{\psi} 4 r_{i n f}}
$$

$\left(\Lambda_{1}^{\prime}\right.$ does not depent on $\left.L_{T}\right)$ into $T_{36}$ and get

$$
T_{36}=0.36 \frac{6}{50.96} \Lambda_{1}^{\prime 2} \frac{L_{\psi}^{2} 16 r^{2}}{q^{4} R^{4}} \frac{1}{\left(1+\frac{Q^{2}}{S^{2}}\right)} q^{2} \frac{1}{\hat{T} v_{T}} \frac{R^{2}}{L_{\psi}^{2}}\left(\frac{\partial \ln T}{\partial x}\right)
$$

or

$$
T_{36}=0.36 \frac{6}{50.96} \Lambda_{1}^{\prime 2} \frac{16 r^{2}}{q^{2} R^{2}} \frac{1}{\left(1+\frac{Q^{2}}{S^{2}}\right)} \frac{1}{\hat{T} v_{T}}\left(\frac{\partial \ln T}{\partial x}\right)
$$

The dependence on the plasma parameters is given by

$$
T_{36}=0.45 q^{2} \frac{R^{2}}{L_{\psi}^{2}} \frac{\nu_{i i, i n f}^{2}}{\Omega^{2}}\left(\hat{T}^{-\frac{5}{2}} \hat{n} \hat{T}^{Z}\right)^{2} \frac{1}{\left(1+\frac{Q^{2}}{S^{2}}\right)} \frac{1}{v_{T}}\left(\frac{\partial \hat{T}}{\partial x}\right)
$$




\section{H Electric drift velocity}

The electric drift speed is given by

$$
-\frac{E_{r}}{B_{\phi}}=v_{E}=v_{\Theta}-\frac{B_{\theta}}{B} v_{\|, i}-v_{N}-\tilde{v}_{T}
$$

We use

$$
\begin{aligned}
g & =\frac{v_{\|, i}}{v_{T}} \\
h & =\frac{v_{\Theta}}{v_{T}}
\end{aligned}
$$

and get the normalized drift speed

$$
\begin{gathered}
\hat{v}_{E}=\frac{v_{E}}{v_{T}}=\frac{v_{\Theta}-\frac{B_{\theta}}{B} v_{\|, i}-v_{N}-\tilde{v}_{T}}{v_{T}} \\
\hat{v}_{E}=h-\frac{B_{\theta}}{B} g-\frac{v_{N}}{v_{T}}-\frac{\tilde{v}_{T}}{v_{T}}
\end{gathered}
$$

The normalized speeds are given by

$$
\begin{gathered}
\frac{\tilde{v}_{T}}{v_{T}}=\frac{\frac{T}{e B} \frac{\partial \ln T}{\partial r}}{\frac{T_{i n f}}{e B L_{\psi}}}=\hat{T} \frac{\partial \ln T}{\partial r} L_{\psi}=\hat{T} \frac{\partial \ln T}{\partial x}=\frac{\partial T}{\partial x} \\
\frac{v_{N}}{v_{T}}=\frac{\frac{T}{e B L_{\psi}} \frac{\partial \ln n}{\partial r}}{\frac{T_{i n f}}{e B}}=\hat{T} \frac{\partial \ln n}{\partial r} L_{\psi}=\hat{T} \frac{\partial \ln n}{\partial x}=\frac{\hat{T}}{\hat{n}} \frac{\partial n}{\partial x}
\end{gathered}
$$




\section{Temperature profile in Alcator C - MOD}

$$
\begin{gathered}
\frac{T_{i}(r)}{T_{i}(0)}=\frac{T_{i}(0)-2 T\left(r_{i n f}\right)}{T_{i}(0)}\left(1-\left[\frac{r}{r_{i n f}}\right]^{2}\right)^{2}+ \\
\frac{T_{i}\left(r_{i n f}\right)}{T_{i}(0)}\left[1-\tanh \frac{r-r_{i n f}}{\Delta_{T}}\right]
\end{gathered}
$$

or

$$
\begin{aligned}
T_{i}(r)= & \left(T_{i}(0)-2 T\left(r_{i n f}\right)\right)\left(1-\left[\frac{r}{r_{i n f}}\right]^{2}\right)^{2}+ \\
& T_{i}\left(r_{i n f}\right)\left[1-\tanh \frac{r-r_{i n f}}{\Delta_{T}}\right]
\end{aligned}
$$

The decaylength at the inversion point is $\Delta_{T}$ again because

$$
\begin{gathered}
\frac{d T_{i}(r)}{d r}=-\left(T_{i}(0)-2 T\left(r_{i n f}\right)\right) 2\left(1-\left[\frac{r}{r_{i n f}}\right]^{2}\right) 2 \frac{r}{r_{i n f}^{2}} \\
\frac{T_{i}\left(r_{i n f}\right)}{\Delta_{T}} \frac{1}{-\cosh ^{2} \frac{r-r_{i n f}}{\Delta_{T}}}
\end{gathered}
$$

at $r=r_{\text {inf }}$ we have

$$
\frac{d T_{i}(r)}{d r}=-\frac{T_{i}\left(r_{i n f}\right)}{\Delta_{T}}
$$

The derivative of the pedestal is given by

$$
\frac{d}{d r} \frac{T_{i}(r)}{T_{i}(0)}=\frac{d}{d r} \frac{T_{i}\left(r_{i n f}\right)}{T_{i}(0)}\left[1-\tanh \frac{r-r_{i n f}}{\Delta_{T}}\right]
$$

and

$$
\left.\frac{d}{d r} \frac{T_{i}(r)}{T_{i}(0)}=\frac{T_{i}\left(r_{i n f}\right)}{T_{i}(0)} \frac{1}{\Delta_{T}}\left[-\frac{1}{\cosh ^{2}\left[\frac{r-r_{i n f}}{\Delta_{T}}\right.}\right]\right]
$$


The logarithmic derivative of the pedestal can be written as

$$
\begin{gathered}
\frac{1}{T_{i}(r)} \frac{d}{d r} T_{i}(r)=T_{i}\left(r_{i n f}\right) \frac{1}{\Delta_{T}}\left[-\frac{1}{\cosh ^{2}\left[\frac{r-r_{i n f}}{\Delta_{T}}\right]}\right] \\
\frac{1}{T_{i}\left(r_{\text {inf }}\right)\left[1-\tanh \frac{r-r_{i n f}}{\Delta_{T}}\right]}
\end{gathered}
$$

or as

$$
\begin{gathered}
\frac{1}{T_{i}(r)} \frac{d}{d r} T_{i}(r)=\frac{1}{\Delta_{T}}\left[-\frac{1}{\cosh ^{2}\left[\frac{r-r_{i n f}}{\Delta_{T}}\right]}\right] \\
\frac{1}{\left[1-\tanh \frac{r-r_{\text {inf }}}{\Delta_{T}}\right]}
\end{gathered}
$$


Forschungszentrum Jülich Jül-3958

Februar 2002

ISSN 0944-2952 